\title{
Astrocytic cell adhesion genes linked to schizophrenia promote synaptic programs in neurons
}

Olli Pietiläinen ${ }^{1,2,3 \# * \dagger}$, Ralda Nehme ${ }^{1,2 \# * \dagger}$, Aditi Trehan ${ }^{1,2}$, Kevin Eggan ${ }^{1,2}$

${ }^{1}$ Stanley Center for Psychiatric Research, Broad Institute of Harvard and MIT, Cambridge, MA 02142, USA

${ }^{2}$ Department of Stem Cell and Regenerative Biology, and the Harvard Institute for Stem Cell Biology, Harvard University, Cambridge, MA 02138, USA

${ }^{3}$ Neuroscience Center, Helsinki Institute for Life Science, University of Helsinki, Helsinki, Finland

\# equal contribution

* correspondence: olli.pietilainen@helsinki.fi, rnehme@broadinstitute.org

$\dagger$ co-senior authors \& supervision of the work

\section{Abstract}

Recent genetic discoveries in schizophrenia have highlighted neuronal genes with functions in the synapse. Although emblematic of neurons, the development of synapses and neuronal maturation relies on interactions with glial cells including astrocytes. To study the role of glia-neuron interactions in schizophrenia, we generated RNA sequence data from human pluripotent stem cell (hPSC) derived neurons that were cocultured with glial cells. We found that expression of genes characteristic of astrocytes induced the expression of post-synaptic genetic programs in neurons, consistent with advanced neuronal maturation. We further found that the astrocyte-induced genes in neurons were associated with risk for schizophrenia. To understand how glial cells promoted neuronal maturation, we studied the association of transcript abundances in glial cells to gene expression in neurons. We found that expression of synaptic cell adhesion molecules in glial cells corresponded to induced synaptic transcripts in neurons and were associated with genetic risk for schizophrenia. These included 11 genes in significant GWAS loci and three with direct genetic evidence for the disorder (MAGI2, NRXN1, LRRC4B, and MSI2). Our results suggest that astrocyte-expressed genes with functions in the synapse are associated with schizophrenia and 
promote synaptic genetic programs in neurons, and further highlight the potential role for astrocyte-neuron interactions in schizophrenia.

\section{Introduction}

Schizophrenia is a severe psychotic disorder characterized by unusual behavior, delusions and hallucinations, flattened motivation, emotion, and interest, as well as impairments in cognitive functioning ${ }^{1}$. Schizophrenia affects $1 \%$ of people globally, but the biological mechanisms underlying the disorder are not well understood ${ }^{2}$. Genetic factors play a major role in schizophrenia etiology, and recent genetic discoveries have highlighted genes that are highly expressed by neurons in the cortical brain and have functions particularly in synapse organization ${ }^{3-5}$. Further mechanistic insight into schizophrenia has emerged with the genetic association of the complement component 4 (C4) and its potential role in developmental synaptic over-pruning detected in schizophrenic brains ${ }^{6}$. Intriguingly, these fundamentally neuronal processes are dependent on interactions with glial cells including astrocytes 7,8 , and raise the question whether non-cell autonomous effects of glial cells on neurons are relevant to schizophrenia.

Astrocytes provide neurons homeostatic support and are important regulators of neuronal development and maturation ${ }^{7}$. They participate in the formation and shaping of the neuronal network, by regulating synapse generation and elimination, transmission, and plasticity. ${ }^{8-10}$ Astrocytes surround neuronal cell bodies and synapses and interact with neurons through a range of contact-dependent and secreted signals that mediate changes in the neuronal maturation state $^{9,11,12}$. However, while we and others have previously shown that glial cells are necessary for the functional maturation of neurons ${ }^{13-16}$, many gaps remain in our understanding of the specific cellular and molecular programs that mediate these processes. To investigate the molecular 
pathways underlying glia-induced neuronal maturation, we performed RNA sequencing of human pluripotent stem cell (hPSC) derived excitatory neurons that were cocultured with mouse glial cells. We reasoned that cellular responses to regulatory interactions between neurons and glia would be in part mediated through mutual changes in transcription that could be detected as correlated gene expression of the cell types. The cross-species cell culture enabled us to separate transcripts by their origin and, therefore, identify molecular dependencies between cocultured neurons and glial cells by studying joint variability in their transcript abundances. Excitingly, our analysis revealed that astrocyte-expressed synaptic cell adhesion molecules, including NRXN1, with established roles in schizophrenia, were associated with induction of synaptic genetic programs in cocultured neurons. These data provide key evidence that the cellular processes in glia that are associated with neuronal maturation involving synaptic programs in vitro are relevant to schizophrenia and provide mechanistic insight into the potential role of astrocytes in psychiatric disorders.

\section{Results}

Assembly of RNA sequencing libraries of hPSC-derived neurons grown with or without glial cells

To study molecular pathways in glia-neuron interactions, we assembled a sample set of 32 karyotypically normal hPSC lines (29 iPSC and three ESC lines) derived from healthy donors (Figure 1, Supplementary table 1). These lines were differentiated into excitatory neurons using a well-characterized protocol that combines Ngn2 expression with forebrain patterning factors to robustly generate a homogenous excitatory neuron population $(\mathrm{N}=32 \text { cell lines })^{16}$. At day four, we plated the neuronal cultures onto a monolayer of glial cells that were prepared as described 
previously ${ }^{17}$ from the cortex of neonatal mice (P1-P3), We have previously shown that co-culture with glia cells is necessary for functional neuronal maturation, which we assessed by measuring synaptically driven network activity using Multielectrode arrays (MEA) ${ }^{16}$. Here, in order to measure the effect of glia-neuron co-culture on gene expression, we allowed five hPSC lines (out of 32) to differentiate in glia-free condition as the negative control for glia-induced outcomes in neurons. We then recorded RNA sequence data from the 33 differentiations at day 28 of the experiment, when the cocultured neurons display robust neuronal morphology and electrophysical activity $^{16}$. To explore differences in developmental trajectories, we also completed RNA sequencing (RNAseq) at day 4 of the differentiation for the same lines (in 34 independent differentiations) prior to glia coculture, when the differentiating cells resembled neuronal progenitor like cells (NPCs), as described previously ${ }^{16,18}$, totaling to 67 RNA libraries (Supplementary table 1). All 67 differentiation were carried out in three experimental replicates each.

\section{Leveraging cross-species co-culture to distinguish RNA molecules from different cell types}

In order to characterize cell-type specific transcriptional effects in the cross-species co-culture, we first confirmed that we could reliably distinguish between RNA molecules that originated from the human neurons and mouse glial-cells ${ }^{19,20}$. To this end, following sequencing, we aligned the reads to a mixed Ensembl human-mouse reference genome (GRCh37/hg19 and GRCm38/mm10, respectively; GSE63269) ${ }^{20}$ and compared these to read counts obtained after aligning to human genome assembly alone (GRCh37/hg19). We used variance partitioning to estimate the proportion of variability in the gene expression estimates from the two alignments. Reassuringly, we found minimal cross-species mapping (Figure 2A-C). For the majority of genes, the differences in the 
alignment added little or no variance to the RNA abundance estimates (average $0.78 \%$ explained of total variance, median $=0 \%$ ). Importantly, only for 329 genes the alignment explained over $10 \%$ of total variance in gene expression (Figure $2 \mathrm{~A}$ ). These 329 genes were on average $22.3 \mathrm{~kb}$ shorter than the rest $(95 \%-\mathrm{CI}: 14.1 \mathrm{~kb}-30.6 \mathrm{~kb} \mathrm{p}=0.00035$, Mann-Whitney) and did not highlight any specific gene ontology (GO) enrichment ( $q>0.05$ for all terms). Expectedly, the alignment to the mixed genome yielded slightly lower (average 3.9\%) library size due to ambiguously mapped reads between the species (average pairwise difference in read counts $=506 \times 10^{3}$ reads (95\%-CI: $\left.765 \times 10^{3}-247 \times 10^{3}\right), p=0.0002$, paired t-test).

A principal component analysis (PCA) in the day 28 neurons and day four NPCs confirmed that the alignment effect was similar in the cocultured and glia-free experiments and did not confound the primary sources of biological variability in the data. Encouragingly, as evident from the superimposed data in PCA, the primary components of gene expression were not affected by the alignment (Figure 2B). Instead, the small fraction of genes whose expression estimates were impacted by cross-species mapping were captured in PC7 and PC8 (explaining 5\% of the total variability) (Figure 2C). Importantly, this was not dependent on the differentiation stage or the presence of glia coculture suggesting that there was no major bias by experimental condition in the read count estimates. Together, these results confirmed that we could accurately separate gene expression data from human neurons and mouse glial cells.

\section{Neurons grown with mouse glia cells exhibit global changes in gene expression}

Having confirmed that we could reliably assign sequence reads to correct cell populations, we next aimed to confirm the neuronal identity of the day 28 neurons (Supplementary Figure 1A). A comparison of the expression of canonical marker genes (Supplementary Table 2) in neurons and 
day 4 NPCs confirmed the expected temporal reduction of the pluripotency genes POU5F1/Oct4 and MKI67 (Neurons grown without glia: $\beta=-1.48$, se: $0.24, p=7.95 \times 10^{-9}$ and Neurons + glia: $\beta$ $=-1.39$, se: $\left.0.13, \mathrm{p}=5.4 \times 10^{-20}\right)$. Expectedly, neuronal identity genes $(D C X, M A P 2, M A P T$, NCAM1, RBFOX3, SYN1, TUBB3) were induced over time in day 28 neurons (both with or without glia co-culture) compared to day 4 NPCs (Neurons without glia: $\beta=0.36$, se: $0.17, p=0.03$; Neurons + glia: $\beta=0.75$, se: $\left.0.09, p=3.1 \times 10^{-16}\right)$. This was accompanied with modest overall reduction in neuronal progenitor markers genes (EMX2, HES1, MSI1, NEUROD1, OTX2; Neurons without glia: $\beta=-0.46$, se: $0.20, p=0.03$; Neurons + glia: $\beta=-0.42$, se: $0.11, p=0.0002$ ), suggesting that the neurons might retain some features of less mature neuronal stages.

We then focused on characterizing the global transcriptome landscape of the differentiating neurons in both culture conditions. Expectedly, the largest component of variation in PCA (PC1: $44 \%$ ) resulted from time in differentiation. Interestingly, within the neuron cluster, the ones in the glia-free condition grouped slightly closer to NPCs than cocultured neurons (Figure 2B, Supplementary Figure 1B). This gradual separation became more prominent in PCA after omitting data from the NPCs (Figure 2D). Taken together, the data suggested that glia coculture induces continuous global transcriptional changes that were consistent with progressive neuronal maturation.

\section{Astrocytes are the predominant cell type in the mouse glial cultures}

Next, we moved on to characterize the glial cell populations in neurons from the 28 lines cocultured with glia by exploring the expression of genes characteristic of specific glia populations from the mouse RNAseq data (Supplementary Table 2). In line with previous work ${ }^{17}$, we observed a predominant expression of genes characteristic of astrocytes compared to marker genes 
for oligodendrocytes $\left(\mathrm{FC}=2.37, \mathrm{p}=2 \times 10^{-30}\right)$, homeostatic oligodendrocytes $(\mathrm{FC}=2.01, \mathrm{p}=6$ $\left.\times 10^{-13}\right)$, and microglia $\left(\mathrm{FC}=3.89, \mathrm{p}=1 \times 10^{-47}\right)$ in the glia coculture (Figure $\left.\mathbf{3 A}\right)$.

Intrigued by astrocytes' role in regulating neuronal maturation ${ }^{21}$, we next wondered whether genetic programs in glial cells that are co-expressed with astrocyte marker genes underlie neuronal maturation in the coculture. For this purpose, we used singular value decomposition to generate a single astrocyte eigengene from six canonical marker genes distinctive of astrocytes (Aldh1l1, Slc1a3, Slc1a2, Gfap, Notch1, S100b) (Figure 3B). The marker genes were highly correlated and the eigengene captured $97 \%$ of the variance. This is consistent with the marker gene transcripts originating from a single shared cell-type ${ }^{22}$ - presumed to be astrocytes - in the glia coculture, and further confirmed the generated eigengene as a relevant proxy for astrocytes in the glial coculture. For glia-free cultures the astrocyte eigengene value was set to zero. This was in agreement with an extrapolated value (0.008) from the astrocyte marker gene expression for the glia free cultures (Figure 3B).

\section{Astrocytes induce transcripts with synaptic functions in neurons}

Next, we investigated whether differences in the astrocyte eigengene value corresponded to gene expression changes in neurons using a multifactorial linear model in the Limma package ${ }^{23}$. Strikingly, the analysis revealed transcript abundances of 4,195 genes (out of 16,694) to be associated with the astrocyte eigengene value (FDR $<5 \%$ ). Out of these, 1,970 transcripts were induced in neurons in cocultures that had high astrocyte eigengene values and 2,225 were reduced. Altogether we found 250 neuronal genes (135 induced, 115 reduced) transcriptome-wide significantly $\left(\mathrm{p}<3.0 \times 10^{-6}\right.$, Bonferroni) associated with the coculture astrocyte eigengene values (Figure 3C, Supplementary table 3). 
We used gene ontology (GO) enrichment analysis to explore whether transcripts in neurons that were induced by high astrocyte eigengene values were enriched for specific biological functions. The analysis of the 135 transcriptome-wide significantly induced neuronal genes revealed most significant enrichments for functions in synapse assembly (GO:0007416, N=9 genes, $\mathrm{FC}=7.03, \mathrm{q}=0.004)$, axon development (GO:0061564, $\mathrm{N}=15$ genes, $\mathrm{FC}=3.5, \mathrm{q}=0.005)$, negative regulation of JAK-STAT cascade (GO:0046426, N=5, FC=13.6, q =0.005), and chemical synaptic signaling (GO:0007268, N=16, $\mathrm{FC}=3.2, \mathrm{q}=0.005)$ (Supplementary Figure2, Supplementary table 4). This suggested that astrocytes in the cocultures induced genetic programs related to synapse biology in the accompanying hPSC derived neurons. Interestingly, among the most frequently included genes in the enriched categories were members encoding for trans-synaptic cell adhesion proteins including neuroligins (NGLN2, NGLN3), NECTIN1, and leucine rich repeat membrane proteins ( $L R R C 4 B, L R R T M 1)$, with roles in anchoring the synaptic nerve terminals. In comparison the 115 transcriptome-wide significantly reduced neuronal transcripts in response to astrocytes did not reveal significant enrichment for any specific biological process. However, extending the GO term analysis to all 2,225 reduced transcripts in neurons with FDR $<5 \%$, there was enrichment for extracellular matrix organization

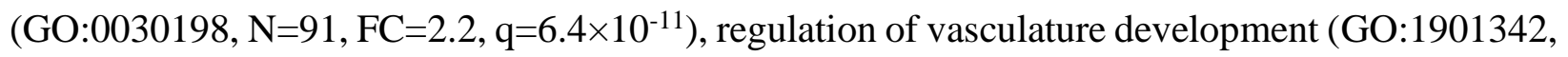
$\mathrm{N}=72$ genes, $\mathrm{FC}=1,8, \mathrm{q}=.3 \times 10^{-4}$ ), as well as leukocyte activation $(\mathrm{GO}: 0002444, \mathrm{~N}=96, \mathrm{FC}=1.6$ $\left.\mathrm{q}=1.1 \times 10^{-3}\right)($ Supplementary table 5$)$.

Encouraged by findings from the GO analysis, we next asked whether the rest, 1,970 induced genes that passed the FDR $<5 \%$ had roles in specific synaptic components in SynGO ${ }^{24}$. We observed a significant enrichment for genes in the presynaptic component (GO:0045202, $\mathrm{N}=$ 98, genes, $\left.\mathrm{q}=8.4 \times 10^{-7}\right)$, and even stronger enrichment for postsynaptic genes $(\mathrm{GO}: 0098794, \mathrm{~N}=$ 
121 genes, $\left.\mathrm{q}=2.9 \times 10^{-8}\right)$, especially in postsynaptic specialization $\left(\mathrm{N}=70\right.$ genes, $\left.\mathrm{q}=3.6 \times 10^{-7}\right)$, and postsynaptic density $\left(\mathrm{N}=51\right.$ genes, $\left.\mathrm{q}=2.1 \times 10^{-4}\right)$ (Figure 3E, Supplementary table 6). These included 23 transcriptome-wide significantly induced neuronal genes, 13 of which were postsynaptic (Figure 3D). Together these results implied that astrocytes in coculture are capable of inducing genetic programs in neurons related to synaptic (particularly postsynapse) biology, which is consistent with synaptic maturation.

\section{Astrocyte expressed genes induce synaptic programs in neurons}

Having established that glia cocultures that have high expression of astrocyte marker genes associate with induction of synaptic gene programs in accompanying neurons, we next wondered whether specific genes in the glial cells were associated with the neuronal response to astrocytes. For this purpose, we first decided to condense the neuronal expression profile of the 1,970 astrocyte-induced transcripts by singular value decomposition to a single eigengene. The generated neuron eigengene values were similar to those of the 135 transcriptome-wide significantly induced genes $\left(r_{\text {median }}=0.65\right)$ and were highly correlated $(r=0.68)$ with the astrocyte eigengene confirming that the neuron eigengene captured relevant variation in gene expression in the neurons (Figure 4A).

Next, we studied the association between the expression levels of individual glial genes (total 11,627 mapped mouse genes) and the maturation state of the cocultured neurons defined by the neuron eigengene value. The analysis revealed transcriptome-wide significant association of transcript levels of 159 glia genes with the neuron eigengene $\left(\mathrm{p}<4.3 \times 10^{-4}\right.$, Bonferroni). Out of these, 123 genes were positively correlated with the neuron eigengene of the more mature neuronal state and 36 were negatively associated with the neuron eigengene values (Figure 4B,

\section{Supplementary table 7).}


In order to gain insight into the likely source cell type of the 159 glia gene transcripts, we explored their expression in a single cell RNAseq atlas of adult mouse brain ${ }^{25}$. Comparison of the expression patterns of the meta cells from the brain atlas revealed that the 123 genes, whose high transcript levels in glia was associated with higher expression of neuron eigengene, were predominantly expressed by astrocytes compared to other glia cell types $\left(\mathrm{p}=6.9 \times 10^{-26}\right.$, t-test) (Figure 4C). In contrast, the 36 negatively associated glia transcripts showed no evidence of preferential expression between the glial cell types in the mouse brain $(\mathrm{p}=0.81)$. Moreover, the 36 transcripts had significantly reduced expression in mouse brain astrocytes compared to the 123 genes that were associated to induced neuron eigengene values ( $\mathrm{p}=0.0035, \mathrm{t}$-test). These results demonstrated that the individual genes associated with synaptic programs in neurons were abundantly expressed by astrocytes in the mouse brain and suggested a role in pathways related to astrocyte-neuron interaction.

Expression of synaptic cell adhesion molecules by glial cells associate with synaptic programs in neurons

We next wondered whether the 159 glia genes would highlight specific neurobiological processes in glial cells that could underlie the interactions with neurons. GO analysis of the 159 glia transcripts revealed an enrichment of genes with synaptic membrane functions $(\mathrm{GO}: 0097060, \mathrm{~N}=$ 13 genes, $\mathrm{FC}=4.4, \mathrm{q}=0.002)$ (Supplementary table 8). Importantly, this enrichment was driven by genes whose high expression in glia induced high expression of synaptic genes in neurons. Reduced expression of only one synaptic gene in glial cells, Eif4ebpl, encoding for Eukaryotic translation initiation factor $4 \mathrm{e}$ was associated with high neuron eigengene values. Encouraged by these results, we explored curated synaptic annotations from SynGO to confirm 19 glia-expressed genes with annotation to synaptic component, out of which 13 were postsynaptic $(q=0.007)$ and 
6 presynaptic members $(q=0.25)$ (Figures 5A\&B, Supplementary table 9). Remarkably, we noticed that many of these genes were synaptic cell adhesion molecules including Nrxn1, Nlgn1, Nlgn3, Lrrc4, and Lrrc4b. Intriguingly, the pre and postsynaptic Nrxn1 and Nlgn1, respectively, have been recently demonstrated to be abundantly present in astrocytes and have likely roles in the astrocyte processes surrounding the synaptic contacts at the tripartite synapse ${ }^{26,27}$. Further evidence for synaptic cell adhesion molecules came from the synaptic scaffold protein Magi2 and Sorcs1 which associate with neuroligins and Nrxn1, respectively, in the synapse ${ }^{28,29}$.

Astrocytes secrete an array of molecules, including neurotransmitters, modulators, and trophic factors which involves regulated exocytosis of synaptic-like vesicles ${ }^{30}$. Interestingly, several of the identified presynaptic genes in the glia had roles in regulating synaptic vesicle cycle in SynGO (Cadps, Nrxn1, Unc13B, and Prkcb), suggesting a potential role for these genes in regulating secretory vesicle cycle in glial cells. In addition, among the most significantly associated genes was the astrocytic low density lipoprotein receptor related protein Lrp4 that has roles in regulating glutamate transmission (Figure 5B $)^{31}$. Together these results suggested that genes encoding for complexes with both pre- and post-synaptic functions and members of the tripartite synapse are present in glia cells and associated with induced expression of synaptic genetic programs in cocultured neurons.

In comparison, the negatively associated genes did not reveal significant enrichment for specific biological processes. Interestingly, however, the most significantly reduced gene encoded for interleukin 6 family neuropoietic cytokine Lif (leukemia inhibitory factor). Among its many roles, Lif promotes self-renewal and neurogenesis in neuronal stem cells, as well progression of astrocyte precursor cells to mature $\mathrm{GFAP}^{+}$astrocytes $^{32-34}$. Therefore, the reduced Lif expression could indicate progression from neuro-/glio-genesis to more mature cell states. This was further 
supported by high expression of Cmmt5, a marker of late astrocytes ${ }^{35}$, which was the most significantly positively associated gene to the neuronal eigengene.

\section{Glial cells induce genetic programs related to schizophrenia in neurons}

Given that recent genetic discoveries have highlighted neuronally expressed genes with functions in synaptic processes in severe psychiatric illnesses, including schizophrenia ${ }^{3,5,36,37}$, we wondered whether these overlapped with those genes that were found to be induced in neurons by astrocytes. To address this question, we calculated gene-wise associations from gene-surrounding variants using summary statistics from a recent genome-wide association study (GWAS) for schizophrenia $^{38}$ in MAGMA ${ }^{39}$. Using the gene-wise associations, we performed a gene-set analysis for the 1,970 genes that were induced by astrocytes in neurons. Excitingly, the analysis revealed significant association for variants nearby the astrocyte induced genes with schizophrenia $\left(\beta=0.13, p=4.5 \times 10^{-6}\right)($ Figure 6A, Supplementary table 10 $)$. We further divided the genes into those that possessed a synaptic annotation in $\mathrm{SynGO}^{24}$ and non-synaptic genes. This revealed that the association to schizophrenia was predominantly - but not exclusively - driven by synaptic genes induced by astrocytes $\left(\beta_{\text {synaptic }}=0.22, p=0.0009 ; \beta_{\text {non-synaptic }}=0.11, p=0.0004\right)$. The increased expression of genes relevant to schizophrenia by astrocytes in neurons suggests that astrocytes could have a non-cell autonomous impact in regulating cellular processes in schizophrenia.

To assess the specificity of the association to schizophrenia, we repeated the analysis for three other central nervous system phenotypes with variable ages of onset: autism spectrum disorder $(\mathrm{ASD})^{40}$, general intelligence ${ }^{41}$, and Alzheimer's disease $(\mathrm{AD})^{42}$. The 1,970 induced neuronal genes showed modest association for ASD $(\beta=0.05, \mathrm{p}=0.02)$ and general intelligence 
$(\beta=0.07, \mathrm{p}=0.009)$, while there was no evidence for association with AD (Figure 6A). As for schizophrenia, the association for intelligence was stronger for genes with synaptic annotations than for non-synaptic genes $\left(\beta_{\text {synaptic }}=0.16, p=0.02 ; \beta_{\text {non-synaptic }}=0.05, p=0.05\right)$. Together these results demonstrated that the presence of astrocytes in glial cultures was associated with dosedependent induction of genetic programs relevant particularly for schizophrenia in the accompanying neurons including synaptic function and neuronal maturation.

\section{Astrocytic genes that induce synaptic programs in neurons are associated to schizophrenia}

The potential role of astrocyte-neuron interactions in schizophrenia prompted us to investigate whether the genes whose high expression in glial cells was linked to synaptic programs in neurons were involved in schizophrenia. We reasoned that since glial cells are central in regulating many of the neuronal processes that have previously been implicated by genetic studies in schizophrenia ${ }^{43}$, disturbances in the glia-neuron regulatory interactions may also be relevant for the disease. Excitingly, a gene set analysis in MAGMA for variants nearby the 123 glial genes, whose high expression was linked to induction of synaptic programs, were significantly associated to schizophrenia $(\beta=0.28, \mathrm{p}=0.00027)$ (Figure 6B, Supplementary table 11$)$. Since a number of these 123 genes had synaptic functions, we further categorized them as synaptic and nonsynaptic based on annotations in $\mathrm{SynGO}^{24}$. Remarkably, the glia-expressed synaptic genes had 4.3-times stronger association to schizophrenia compared to the non-synaptic genes $\left(\beta_{\text {synaptic }}=\right.$ $\left.0.76, \mathrm{p}=0.0027, \beta_{\text {non-synaptic }}=0.17, \mathrm{p}=0.059\right)($ Figure 6B $)$.

In order to investigate individual genes underlying the gene-set association for schizophrenia, we ranked the 123 glia genes according to the Magma Z-statistic of gene-wise associations. (Figure 6C, Supplementary table 12). Excitingly, the genes with the strongest associations included a number of the synaptic genes including the cell adhesion molecules 
LRRC4, LRRC4B and NRXN1 as well as the astrocytic LRP4 involved in regulation of the glutamate transmission ${ }^{31}$. At this time, a new schizophrenia GWAS from The Psychiatric Genomics Consortium (PGC3) was released reporting 270 loci for schizophrenia³ . Remarkably, 11 of the most significant gene-wise associations of the 123 glia expressed genes were within genome-wide significant loci in PGC3. In addition, two genes had direct genetic evidence from rare variant associations (NRXN1 and MAGI2) $)^{5,44,45}$ and two had been previously implicated by fine mapping of significant GWAS loci $(L R R C 4 B \text { and } M S I 2)^{3}$. Here, we show that these genes' expression in glial cells is associated with synaptic programs and induction of genes linked with schizophrenia in neurons. This further suggests that the neurobiological processes taking place in neuron-glia interactions in vitro are relevant for schizophrenia biology.

\section{Replication of the glia-induced effects}

Next, to validate transcriptional findings for the joint variability in gene expression in cocultured neurons and glial cells, we analyzed RNAseq data from an additional 22 neuronal lines (20 cocultured with glial cells, Supplementary table 1) that were processed together with the discovery data set (Nehme \& Pietilainen et al 2021). The iPSC lines were reprogrammed from 20 donors carrying a $1.5-3 \mathrm{Mb}$ deletion of chromosome $22 \mathrm{q} 11.2^{46}$ which is associated with 68 -fold risk for schizophrenia and is among the highest known risk factors for the disorder ${ }^{47}$. We first characterized the transcriptome data by a joint PCA with the discovery cohort. The analysis clustered the two data sets together in the primary components with PC4 separating the glia-free neurons from the rest of the samples (Figure 7A). This suggested that the patient and control lines behave similarly in the cortical differentiation protocol, in line with previous reports (Nehme \& Pietilainen et al 2021). 
We have previously shown that the deletion neurons were not different from controls in their neuronal identity but were associated with transcriptional changes related to presynaptic programs (Nehme \& Pietilainen et al 2021). We therefore asked whether the transcriptional differences reported previously between neurons from deletion carriers and controls overlapped with genes in neurons associated with cocultured astrocytes in the discovery cohort. We found no evidence that the reported transcriptional differences between the deletion and control neurons Nehme \& Pietilainen et al 2021) resulted from differences in the impact of glial cells on the neurons. Of the 1,970 neuronal genes induced by glial cells, 110 were found nominally significantly changed between deletion patients and controls, which is not different from random sampling ( $\mathrm{p}=1$, binomial test for overlap of differentially expressed genes).

After confirming that the deletion neurons were not different from the control neurons in transcript abundances that were associated with astrocyte presence in the discovery cohort, we proceeded to investigate the association between gene expression in glial cells and neurons in the deletion lines. In an attempt to replicate the findings from the discovery cohort, we first generated a neuron eigengene from the deletion cohort using the same 1,970 genes that were induced by astrocyte presence in the discovery data set. Encouragingly, we found that majority of the 1,970 genes were moderately to highly correlated with the neuron eigengene $\left(r_{\text {median }}=0.41, \mathrm{r}_{\mathrm{Q} 3}=0.77\right)$ in the patient lines (Figure 7B). This suggested that genes that were found to be associated with astrocytes in the discovery cohort, were also co-regulated in the deletion neurons. Additionally, a comparison of the astrocyte marker eigengene values revealed no systematic difference between discovery and deletion data sets $(\mathrm{p}=0.58$, Kolmogorov-Smirnov test), confirming overall similar coculture conditions in the two experimental sets. 
We then asked whether the neuron eigengene was affected by the expression of astrocyte marker genes in the deletion lines. Remarkably, a linear model between the astrocyte markers and neuron eigengene revealed a significant association $(\beta=0.07, \mathrm{SE}=0.03, \mathrm{p}=0.029)$, replicating the findings from the discovery cohort (Figure 7C).

In order to further validate the astrocyte effect on individual gene expression in neurons, we performed a joint analysis in the two data sets for astrocyte eigengene association with neuron gene expression. The results from the joint analysis were highly identical to the ones observed in the discovery cohort alone ( $\mathrm{r}=0.91$ for gens FDR $<5 \%$ in the discovery cohort). Importantly, $75 \%$ of the transcriptome-wide significant neuron genes in the discovery cohort were in the top $10 \%$ of ranked results in the joint analysis (Figure 7D, Supplementary table 13).

We next compared the association of the individual 159 glial genes to the neuron eigengene in the deletion lines. Remarkably, we found that the 159 genes had highly similar - albeit smaller - effect sizes in the deletion lines compared to the discovery cohort $(r=0.8)$. In addition, due to winner's curse and the reduced power of the smaller sample size, the deletion lines revealed overall weaker associations than observed for the discovery cohort (only 11 transcriptome wide significant genes $\left.\mathrm{p}<4 \times 10^{-6}\right)$. However, 37 out of the 159 glia genes from the discovery cohort were nominally significant $(\mathrm{p}<0.05)$ also in the deletion lines with same direction of effect as in the discovery cohort (Expected 6 genes, $\mathrm{p}=7.1 \times 10^{-20}$, binomial test) (Figure 7E, Supplementary table 14). Together, these results provided further validation for the observed associations with glia-genes and synaptic programs in neurons and confirmed that the transcriptional effects associated with the 22q11.2 deletion genotype did not result from a differential neuronal response to glial cells.

\section{Discussion}


Here, we studied how glial cells impact neuronal cell state and maturation by analyzing the co-expression of genes between the two cell populations in coculture using cross-species-resolved RNAseq data. Our analysis revealed that genetic programs in glial cells that covary in abundance with astrocyte markers induce pre- and post-synaptic programs in neurons that associate with schizophrenia (Figures 3 \& 6). We discovered that the neuronal synaptic gene programs were associated with high expression of astrocytic synaptic cell adhesion molecules including neurexins (Nrxn1), neuroligins (Nlgn1, Nlgn3), and leucine rich repeat transmembrane proteins (Lrrc4, $\operatorname{Lrc} 4 b$ ) with direct evidence from both common and rare variants association to schizophrenia ${ }^{3,5}$ (Figures 4-6). Our analysis revealed associations with additional synaptic genes expressed by astrocytes with functions related to synaptic vesicle cycle (Cadps, Nrxn1, Unc13B, and Prkcb), as well as glutamate receptor subunit Gria1 and synaptic potassium channels Kcnb1.

Schizophrenia associations are concentrated in genes that are highly expressed by neurons and cortical regions of the brain with roles in the synapse, transmission and differentiation ${ }^{3-5}$. This has focused much of the previous attention in the field on neurons. Besides neurons, astrocytes are active modulatory components of neural circuits that shape the structure and function of neuronal synapses and ultimately behavior through direct contacts and secreted factors ${ }^{48}$. Indeed, together with the presynaptic and postsynaptic nerve terminals the synaptically associated astrocytes compose a solid functional unit known as a tripartite synapse ${ }^{49}$. Importantly, astrocytes express many gene products typically described as neuronal synaptic elements, including receptors for neurotransmitters, synaptic cell adhesion molecules, and components of synaptic-like vesicle cycle $26,27,30,50$ where gene variants have been associated with risk to schizophrenia ${ }^{3-5,45}$.

Contact between astrocytes and neurons is required for synapse formation ${ }^{51}$. Transsynaptic cell adhesion molecules, such as neurexins (NRXN), neuroligins (NLGN) and leucine 
rich repeat transmembrane protein (LRRTM) provide a structural scaffold that holds synaptic terminals together and participate from early steps of synapse formation to regulating synaptic plasticity $^{52,53}$. Astrocytes have been recently demonstrated to express NRXN1 and NLGN1 implying that they could be involved in fastening also astrocyte processes to the synapse ${ }^{26,27}$. Postsynaptic LRRC4 and LRRC4B associate with postsynaptic density (PSD95) to regulate excitatory synapse formation by LRRC4 binding to presynaptic netrin-G2 (NTNG2) and LRRC4B binding to receptor tyrosine phosphatases LAR (PTPRF), PTPRS, and PTPRD to induce functional presynaptic differentiation in contacting neurites ${ }^{54-56}$. In a manner similar to transsynaptic binding of NRXN1 and NLGN1 ${ }^{57}$, the heterophilic connections with LRRC4 and LRRC4B to their binding partners enable correct connectivity between presynaptic and postsynaptic terminals ${ }^{54,55}$. Here we report that expression of these genes encoding for synaptic cell adhesion molecules in glial cells correlates with the expression of genes with roles in synaptic organization including structural trans-synaptic adhesion molecules in neurons (Figure 3). Importantly, we found that NTNG2 and PTPRS that bind LRRC4 and LRRC4B, respectively, were induced in neurons in response to astrocytes. These findings provide further evidence that these molecules participate in glia-neuron interactions that may involve synaptic contacts. The expression of heterophilic synaptic cell adhesion molecules characteristic for presynaptic and postsynaptic nerve terminals in the glial cells suggest that they may be involved in positioning glial processes with connections to the two neuronal synaptic terminals.

Astrocytes are central in regulating many of the key neuronal functions, related to development, synaptogenesis, maturation, and synaptic transmission that are fundamental to all information processing in the brain ${ }^{7}$. Together with the discoveries of synaptic gene variants in schizophrenia there has been increasing interest in astrocytes' potential role in the disorder ${ }^{43,58}$. 
Schizophrenia is considered to be a neurodevelopmental disorder where the disease pathology initiates years before the onset of clinical symptoms ${ }^{59}$. The neurodevelopmental model is supported by biological and epidemiological data of early pre- and post-natal risk factors, as well as psychiatric symptoms preceding the diagnosis ${ }^{59}$. Previously, regulatory variants in complement component $4(\mathrm{C} 4)$ and their role in developmental synaptic pruning have been linked with schizophrenia ${ }^{6}$. Complement proteins are expressed by neurons and glial cells including astrocytes and are localized to a subset of developing synapses ${ }^{60,61}$. Defects in developmental pruning can lead to synaptic loss long before onset of clinical symptoms in line with the developmental model. Here we show that early developmental glia interactions with hPSC derived neurons with resemblance to late prenatal stages ${ }^{16}$ are associated with coregulated expression of synaptic adhesion genes linked with schizophrenia.

Follow up studies of recent genetic discoveries comparing gene expression patterns across tissues and cell types have implicated neurons as relevant cell type for schizophrenia ${ }^{3-5}$. However, many emblematic neuronal functions are dependent on interplay with non-neuronal glial cells and may impact the pathology through direct or non-cell autonomous effects on neurons. Here we show that expression of schizophrenia-associated genes in glial cells is correlated with a neuronal maturation state and induction of the expression of risk genes in neurons. Our results underscore the importance of evaluating the converging functional impact of emerging genetic discoveries in living biological model systems that provide mechanistic insight, such as cellular interactions, into affected biological processes.

\section{Author contributions}


The project was conceived and designed by OP, RN, and KE. The work was supervised by OP and RN. All experiments were designed by RN and performed by RN and AT (under the supervision of RN). All analyses were conceived, designed, and performed by OP. OP wrote the initial draft of the manuscript and figures. $\mathrm{RN}$, OP and $\mathrm{KE}$ contributed to editing of the manuscript.

\section{Acknowledgements}

We would like to warmly thank the voluntary participants who have donated the cell lines for research via the Swedish Schizophrenia Cohort (Karolinska Institute), the Northern Finnish Intellectual Disability Cohort (NFID), Umea University, Massachusetts General Hospital (MGH), McLean Hospital, and GTEx. This work was funded by U01MH105669 (NIH/NIMH) and the

Stanley Center for Psychiatric Research at the Broad Institute, with additional support from R37NS083524 and U01MH115727. RN was also supported by a NARSAD young investigator award (Brain and Behavior Research Foundation). OP was additionally supported by Instrumentarium Science Foundation, Sigrid Juselius Foundation, Orion Research Foundation, Jalmari and Rauha Ahokas Foundation, Päivikki and Sakari Sohlberg Foundation, Jenny and Antti Wihuri foundation, and Niilo Helander Foundation.

\section{References}

1 van Os, J. \& Kapur, S. Schizophrenia. Lancet 374, 635-645, doi:10.1016/S01406736(09)60995-8 (2009).

2 Owen, M. J., Sawa, A. \& Mortensen, P. B. Schizophrenia. Lancet 388, 86-97, doi:10.1016/S0140-6736(15)01121-6 (2016).

3 Ripke, S., Walters, J. T. \& O’Donovan, M. C. Mapping genomic loci prioritises genes and implicates synaptic biology in schizophrenia. medRxiv 2020.09.12.20192922, doi:doi: https://doi.org/10.1101/2020.09.12.20192922 (2020). 
4 Finucane, H. K. et al. Partitioning heritability by functional annotation using genomewide association summary statistics. Nat Genet 47, 1228-1235, doi:10.1038/ng.3404 (2015).

5 Singh, T., Neale, B. M. \& Daly, M. J. Exome sequencing identifies rare coding variants in 10 genes which confer substantial risk for schizophrenia. medRxiv

2020.09.18.20192815, doi:doi: https://doi.org/10.1101/2020.09.18.20192815 (2020).

6 Sekar, A. et al. Schizophrenia risk from complex variation of complement component 4. Nature 530, 177-183, doi:10.1038/nature16549 (2016).

7 Verkhratsky, A. \& Nedergaard, M. Physiology of Astroglia. Physiol Rev 98, 239-389, doi:10.1152/physrev.00042.2016 (2018).

8 Eroglu, C. \& Barres, B. A. Regulation of synaptic connectivity by glia. Nature 468, 223231, doi:10.1038/nature09612 (2010).

9 Clarke, L. E. \& Barres, B. A. Emerging roles of astrocytes in neural circuit development. Nat Rev Neurosci 14, 311-321, doi:10.1038/nrn3484 (2013).

10 Pfrieger, F. W. Roles of glial cells in synapse development. Cell Mol Life Sci 66, 20372047, doi:10.1007/s00018-009-0005-7 (2009).

11 Allen, N. J. \& Eroglu, C. Cell Biology of Astrocyte-Synapse Interactions. Neuron 96, 697-708, doi:10.1016/j.neuron.2017.09.056 (2017).

12 Allen, N. J. Astrocyte regulation of synaptic behavior. Annu Rev Cell Dev Biol 30, 439463, doi:10.1146/annurev-cellbio-100913-013053 (2014).

13 Pfrieger, F. W. \& Barres, B. A. Synaptic efficacy enhanced by glial cells in vitro. Science 277, 1684-1687, doi:10.1126/science.277.5332.1684 (1997).

14 Ullian, E. M., Sapperstein, S. K., Christopherson, K. S. \& Barres, B. A. Control of synapse number by glia. Science 291, 657-661, doi:10.1126/science.291.5504.657 (2001).

15 Vierbuchen, T. et al. Direct conversion of fibroblasts to functional neurons by defined factors. Nature 463, 1035-1041, doi:10.1038/nature08797 (2010).

16 Nehme, R. et al. Combining NGN2 Programming with Developmental Patterning Generates Human Excitatory Neurons with NMDAR-Mediated Synaptic Transmission. Cell Rep 23, 2509-2523, doi:10.1016/j.celrep.2018.04.066 (2018).

17 Di Giorgio, F. P., Boulting, G. L., Bobrowicz, S. \& Eggan, K. C. Human embryonic stem cell-derived motor neurons are sensitive to the toxic effect of glial cells carrying an ALScausing mutation. Cell Stem Cell 3, 637-648, doi:10.1016/j.stem.2008.09.017 (2008).

18 Wells, M. F. et al. Genome-wide screens in accelerated human stem cell-derived neural progenitor cells identify Zika virus host factors and drivers of proliferation. bioRxiv 476440, doi:doi: https://doi.org/10.1101/476440 (2018).

19 Burke, E. E. et al. Dissecting transcriptomic signatures of neuronal differentiation and maturation using iPSCs. Nat Commun 11, 462, doi:10.1038/s41467-019-14266-z (2020).

20 Macosko, E. Z. et al. Highly Parallel Genome-wide Expression Profiling of Individual Cells Using Nanoliter Droplets. Cell 161, 1202-1214, doi:10.1016/j.cell.2015.05.002 (2015).

21 Tang, X. et al. Astroglial cells regulate the developmental timeline of human neurons differentiated from induced pluripotent stem cells. Stem Cell Res 11, 743-757, doi:10.1016/j.scr.2013.05.002 (2013). 
22 Zaitsev, K., Bambouskova, M., Swain, A. \& Artyomov, M. N. Complete deconvolution of cellular mixtures based on linearity of transcriptional signatures. Nat Commun 10, 2209, doi:10.1038/s41467-019-09990-5 (2019).

23 Law, C. W., Chen, Y., Shi, W. \& Smyth, G. K. voom: Precision weights unlock linear model analysis tools for RNA-seq read counts. Genome Biol 15, R29, doi:10.1186/gb2014-15-2-r29 (2014).

24 Koopmans, F. et al. SynGO: An Evidence-Based, Expert-Curated Knowledge Base for the Synapse. Neuron 103, 217-234 e214, doi:10.1016/j.neuron.2019.05.002 (2019).

25 Saunders, A. et al. Molecular Diversity and Specializations among the Cells of the Adult Mouse Brain. Cell 174, 1015-1030 e1016, doi:10.1016/j.cell.2018.07.028 (2018).

26 Trotter, J. H. et al. Astrocytic Neurexin-1 Orchestrates Functional Synapse Assembly. BioRxiv 2020.08.21.262097, doi:doi: https://doi.org/10.1101/2020.08.21.262097 (2020).

27 Stogsdill, J. A. et al. Astrocytic neuroligins control astrocyte morphogenesis and synaptogenesis. Nature 551, 192-197, doi:10.1038/nature24638 (2017).

28 Sumita, K. et al. Synaptic scaffolding molecule (S-SCAM) membrane-associated guanylate kinase with inverted organization (MAGI)-2 is associated with cell adhesion molecules at inhibitory synapses in rat hippocampal neurons. J Neurochem 100, 154-166, doi:10.1111/j.1471-4159.2006.04170.x (2007).

29 Ribeiro, L. F. et al. SorCS1-mediated sorting in dendrites maintains neurexin axonal surface polarization required for synaptic function. PLoS Biol 17, e3000466, doi:10.1371/journal.pbio.3000466 (2019).

30 Verkhratsky, A., Matteoli, M., Parpura, V., Mothet, J. P. \& Zorec, R. Astrocytes as secretory cells of the central nervous system: idiosyncrasies of vesicular secretion. EMBO J 35, 239-257, doi:10.15252/embj.201592705 (2016).

31 Sun, X. D. et al. Lrp4 in astrocytes modulates glutamatergic transmission. Nat Neurosci 19, 1010-1018, doi:10.1038/nn.4326 (2016).

32 Pitman, M. et al. LIF receptor signaling modulates neural stem cell renewal. Mol Cell Neurosci 27, 255-266, doi:10.1016/j.mcn.2004.07.004 (2004).

33 Koblar, S. A. et al. Neural precursor differentiation into astrocytes requires signaling through the leukemia inhibitory factor receptor. Proc Natl Acad Sci U S A 95, 3178-3181, doi:10.1073/pnas.95.6.3178 (1998).

34 Bauer, S., Kerr, B. J. \& Patterson, P. H. The neuropoietic cytokine family in development, plasticity, disease and injury. Nat Rev Neurosci 8, 221-232, doi:10.1038/nrn2054 (2007).

35 Tiwari, N. et al. Stage-Specific Transcription Factors Drive Astrogliogenesis by Remodeling Gene Regulatory Landscapes. Cell Stem Cell 23, 557-571 e558, doi:10.1016/j.stem.2018.09.008 (2018).

36 Mullins, N. et al. Genome-wide association study of more than 40,000 bipolar disorder cases provides new insights into the underlying biology. Nat Genet, doi:10.1038/s41588021-00857-4 (2021).

37 Palmer, D. S. et al. Exome sequencing in bipolar disorder reveals shared risk gene AKAP11 with schizophrenia. medRxiv 2021.03.09.21252930, doi:doi: https://doi.org/10.1101/2021.03.09.21252930 (2021).

38 Schizophrenia Working Group of the Psychiatric Genomics, C. Biological insights from 108 schizophrenia-associated genetic loci. Nature 511, 421-427, doi:10.1038/nature13595 (2014). 
39 de Leeuw, C. A., Mooij, J. M., Heskes, T. \& Posthuma, D. MAGMA: generalized geneset analysis of GWAS data. PLoS Comput Biol 11, e1004219, doi:10.1371/journal.pcbi.1004219 (2015).

40 Autism Spectrum Disorders Working Group of The Psychiatric Genomics, C. Metaanalysis of GWAS of over 16,000 individuals with autism spectrum disorder highlights a novel locus at 10q24.32 and a significant overlap with schizophrenia. Mol Autism 8, 21, doi:10.1186/s13229-017-0137-9 (2017).

41 Savage, J. E. et al. Genome-wide association meta-analysis in 269,867 individuals identifies new genetic and functional links to intelligence. Nat Genet 50, 912-919, doi:10.1038/s41588-018-0152-6 (2018).

42 Jansen, I. E. et al. Genome-wide meta-analysis identifies new loci and functional pathways influencing Alzheimer's disease risk. Nat Genet 51, 404-413, doi:10.1038/s41588-018-0311-9 (2019).

43 Goudriaan, A. et al. Specific glial functions contribute to schizophrenia susceptibility. Schizophr Bull 40, 925-935, doi:10.1093/schbul/sbt109 (2014).

44 Stefansson, H. et al. Large recurrent microdeletions associated with schizophrenia. Nature 455, 232-236, doi:10.1038/nature07229 (2008).

45 Rujescu, D. et al. Disruption of the neurexin 1 gene is associated with schizophrenia. Hum Mol Genet 18, 988-996, doi:10.1093/hmg/ddn351 (2009).

46 Edelmann, L., Pandita, R. K. \& Morrow, B. E. Low-copy repeats mediate the common 3$\mathrm{Mb}$ deletion in patients with velo-cardio-facial syndrome. Am J Hum Genet 64, 10761086, doi:10.1086/302343 (1999).

47 Marshall, C. R. et al. Contribution of copy number variants to schizophrenia from a genome-wide study of 41,321 subjects. Nat Genet 49, 27-35, doi:10.1038/ng.3725 (2017).

48 Araque, A. et al. Gliotransmitters travel in time and space. Neuron 81, 728-739, doi:10.1016/j.neuron.2014.02.007 (2014).

49 Araque, A., Parpura, V., Sanzgiri, R. P. \& Haydon, P. G. Tripartite synapses: glia, the unacknowledged partner. Trends Neurosci 22, 208-215, doi:10.1016/s01662236(98)01349-6 (1999).

50 Agulhon, C. et al. What is the role of astrocyte calcium in neurophysiology? Neuron 59, 932-946, doi:10.1016/j.neuron.2008.09.004 (2008).

51 Barker, A. J., Koch, S. M., Reed, J., Barres, B. A. \& Ullian, E. M. Developmental control of synaptic receptivity. J Neurosci 28, 8150-8160, doi:10.1523/JNEUROSCI.174408.2008 (2008).

52 McAllister, A. K. Dynamic aspects of CNS synapse formation. Annu Rev Neurosci 30, 425-450, doi:10.1146/annurev.neuro.29.051605.112830 (2007).

53 Farhy-Tselnicker, I. \& Allen, N. J. Astrocytes, neurons, synapses: a tripartite view on cortical circuit development. Neural Dev 13, 7, doi:10.1186/s13064-018-0104-y (2018).

54 Kim, S. et al. NGL family PSD-95-interacting adhesion molecules regulate excitatory synapse formation. Nat Neurosci 9, 1294-1301, doi:10.1038/nn1763 (2006).

55 Woo, J. et al. Trans-synaptic adhesion between NGL-3 and LAR regulates the formation of excitatory synapses. Nat Neurosci 12, 428-437, doi:10.1038/nn.2279 (2009).

56 Kwon, S. K., Woo, J., Kim, S. Y., Kim, H. \& Kim, E. Trans-synaptic adhesions between netrin-G ligand-3 (NGL-3) and receptor tyrosine phosphatases LAR, protein-tyrosine phosphatase delta (PTPdelta), and PTPsigma via specific domains regulate excitatory 
synapse formation. J Biol Chem 285, 13966-13978, doi:10.1074/jbc.M109.061127 (2010).

57 Graf, E. R., Zhang, X., Jin, S. X., Linhoff, M. W. \& Craig, A. M. Neurexins induce differentiation of GABA and glutamate postsynaptic specializations via neuroligins. Cell 119, 1013-1026, doi:10.1016/j.cell.2004.11.035 (2004).

58 Dietz, A. G., Goldman, S. A. \& Nedergaard, M. Glial cells in schizophrenia: a unified hypothesis. Lancet Psychiatry 7, 272-281, doi:10.1016/S2215-0366(19)30302-5 (2020).

59 Rapoport, J. L., Giedd, J. N. \& Gogtay, N. Neurodevelopmental model of schizophrenia: update 2012. Mol Psychiatry 17, 1228-1238, doi:10.1038/mp.2012.23 (2012).

60 Stevens, B. et al. The classical complement cascade mediates CNS synapse elimination. Cell 131, 1164-1178, doi:10.1016/j.cell.2007.10.036 (2007).

61 Stephan, A. H., Barres, B. A. \& Stevens, B. The complement system: an unexpected role in synaptic pruning during development and disease. Annu Rev Neurosci 35, 369-389, doi:10.1146/annurev-neuro-061010-113810 (2012). 
Figure 1. Outline of the experimental design. Neuronal induction with $\mathrm{Ngn} 2$ and small molecule patterning was conducted for hPSCs from 34 healthy donors. At day 4 of the differentiation part of the neuronal cultures were plated on mouse glial cells and others were left to differentiate alone. RNAseq was performed at day 28 of the differentiation and the libraries were resolved by species followed by analysis of normalized read counts.
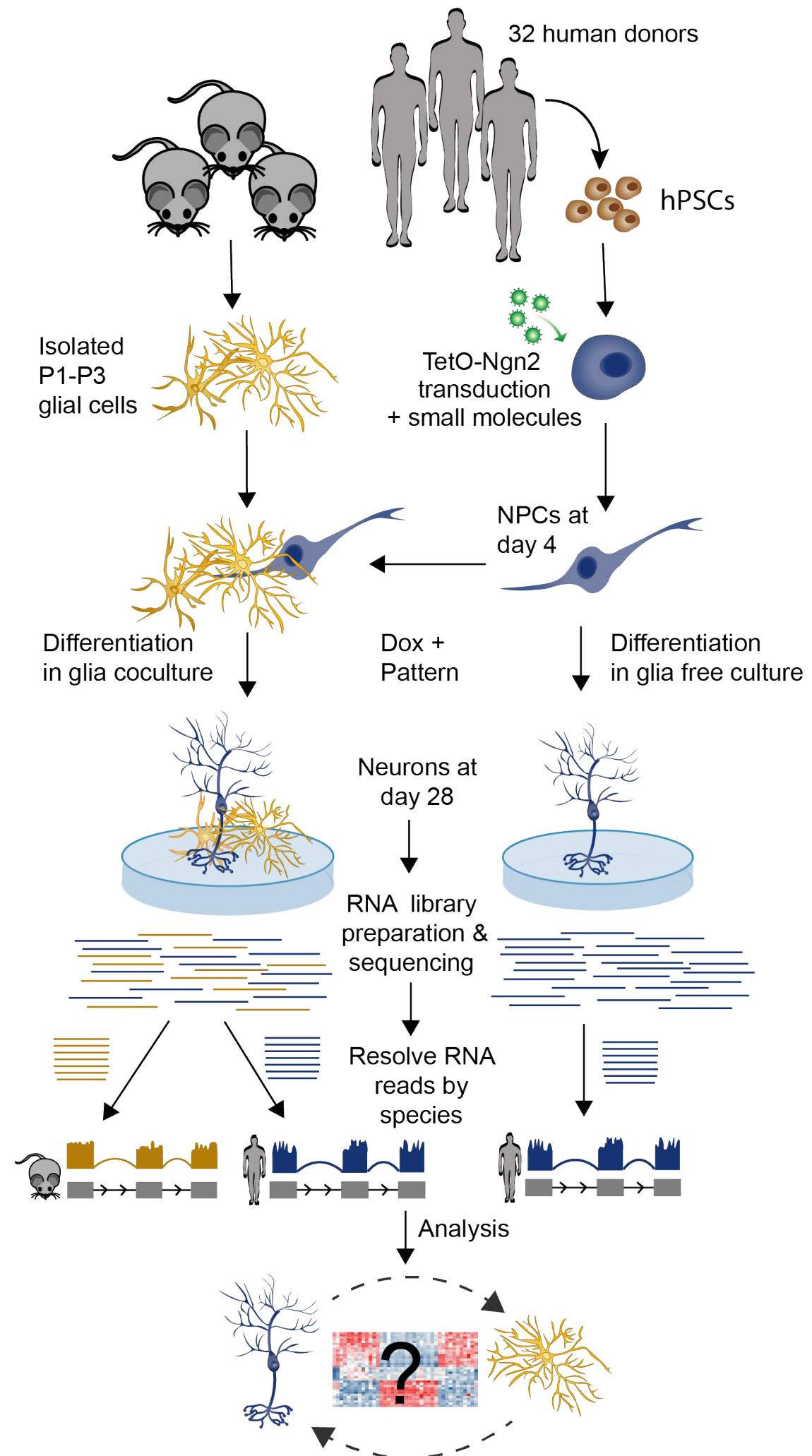
Figure 2. Characterization of the transcriptome of human neurons. A) Variance partitioning by human and mixed reference genome reveals 329 genes for which cross-species mapping explains $>10 \%$ of variation in read counts. On average cross-species mapping explains only $0.78 \%$ of the variation in the read counts. B\& C) PCA of RNAseq data from day 4 NPCs and day 28 neurons aligned to human and mixed reference genomes. D) PCA of RNAseq data from day 28 neurons aligned to mixed refence genome.

A Majority of genes are not impacted by cross-species mapping (Avg. $0 \%$ of variance)

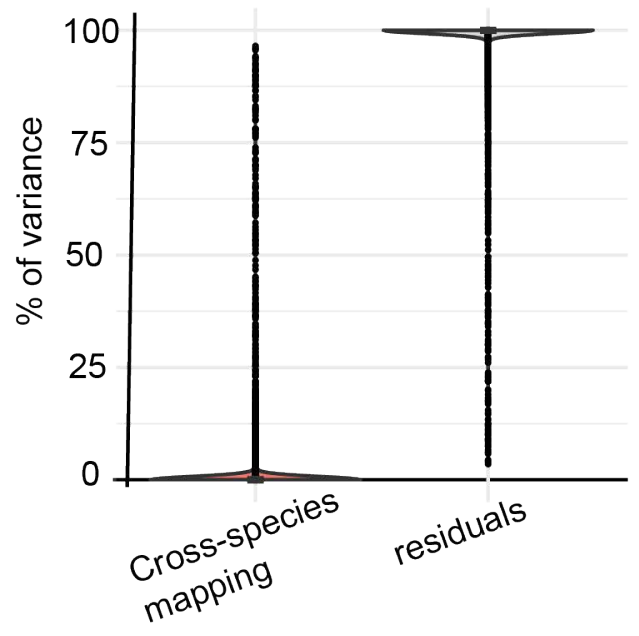

PCs $7 \& 8$ capture variance from cross-species mapping

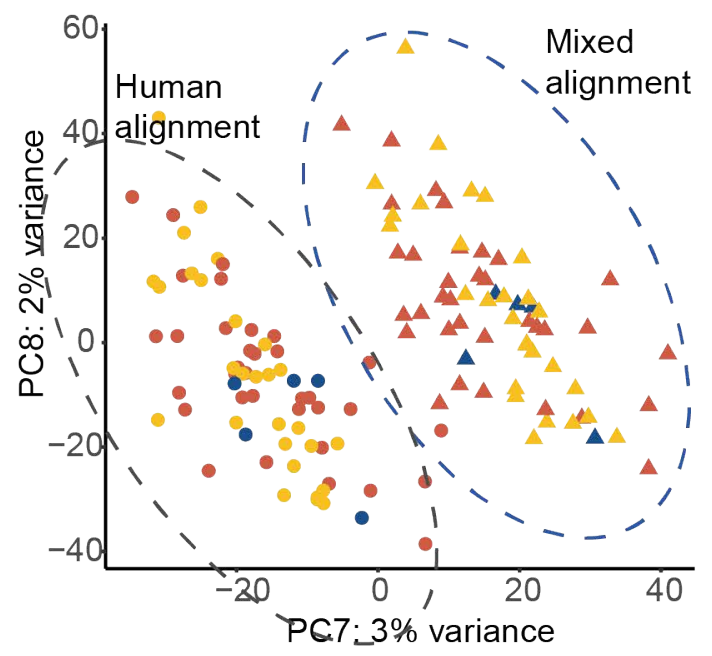

B PC1 captures changes due to time in differentiation

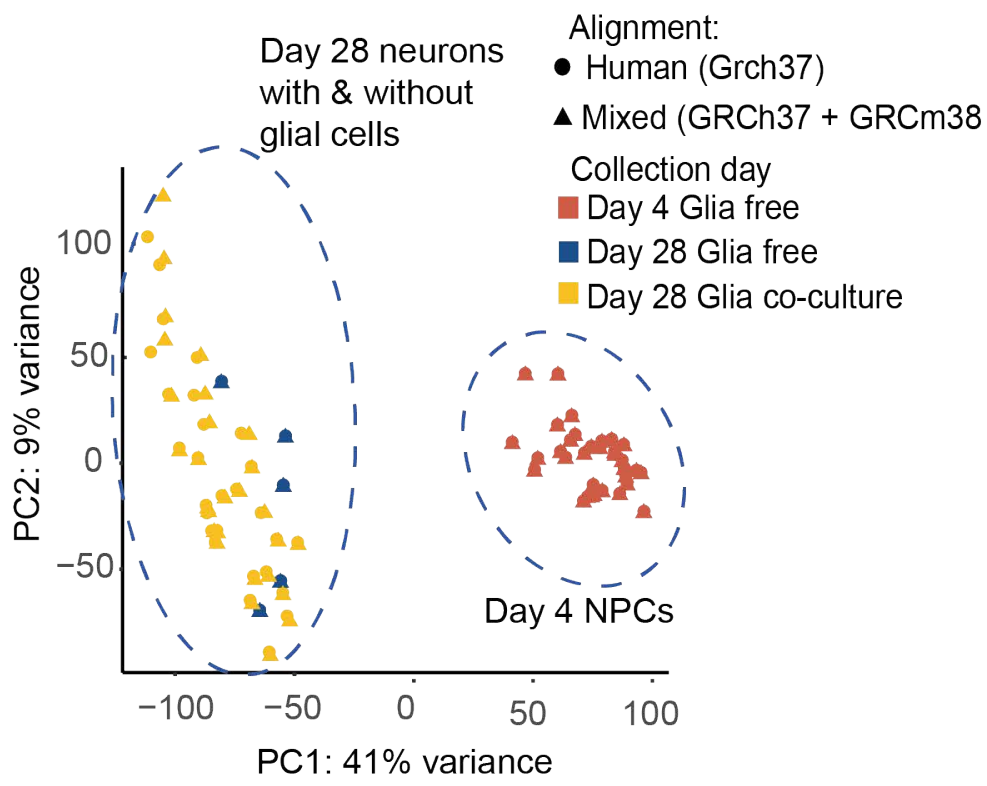

D PCA for RNASeq from day 28 neurons capture variance related to culuture condtion

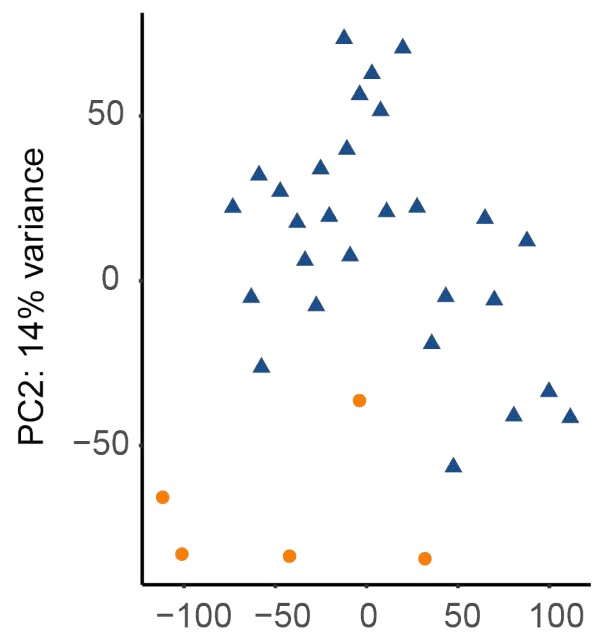

Glia coculture
- no
$\Delta$ yes

Collection Day Day 4

- Day 28 
Figure 3. Astrocyte eigengene associates with synaptic gene expression in cocultured neurons. A) Expression

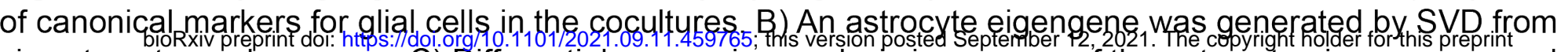

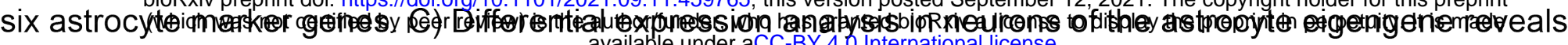

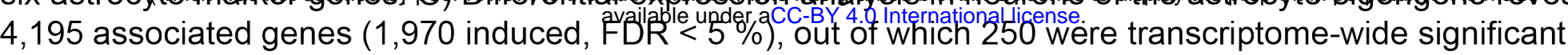
$(p<3.0 \times 10-6$, Bonferroni, colored genes). D) Synaptic genes were induced in neurons in cocultures with high expression of astrocyte marker genes. E) Astrocyte induced genes in neurons were enriched for synaptic annotations in SynGO.

A Expression of canonical glial cell marker genes in glia coculutures

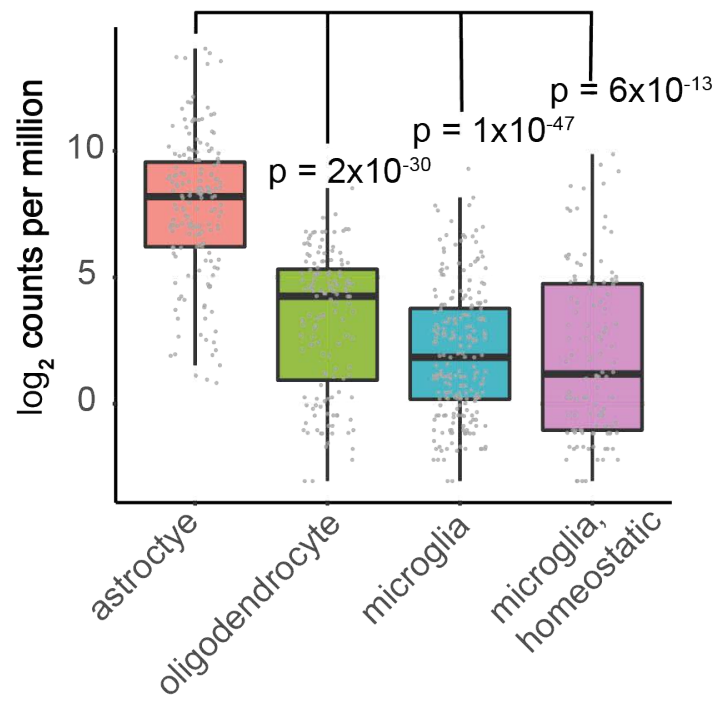

C Astrocyte eigengene associates with global

transcriptional changes in coculutred neurons

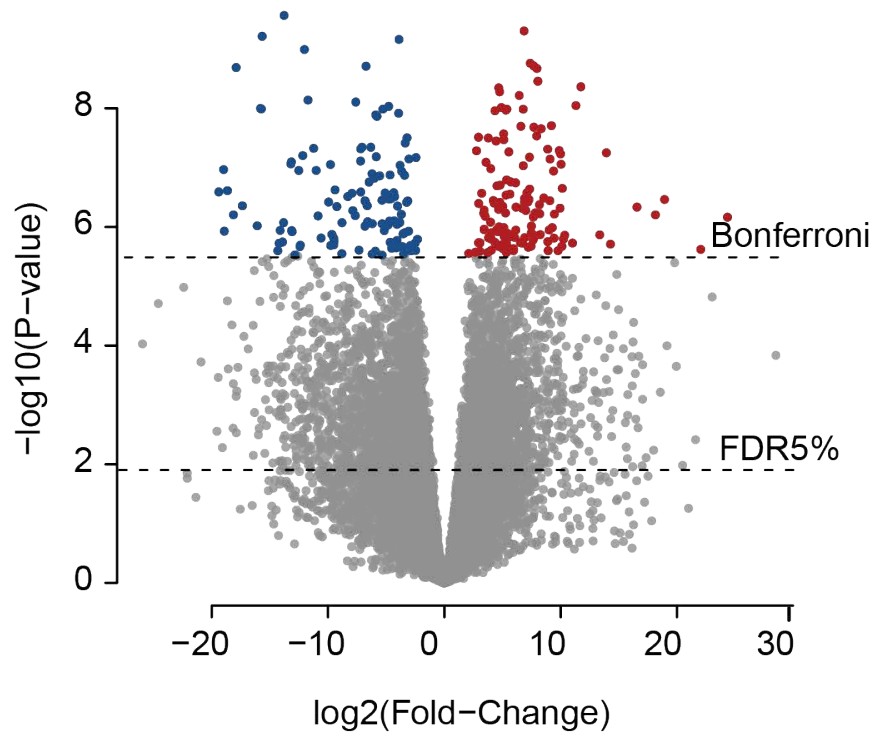

B Astrocyte marker genes are highly correlated with the generated eigengene

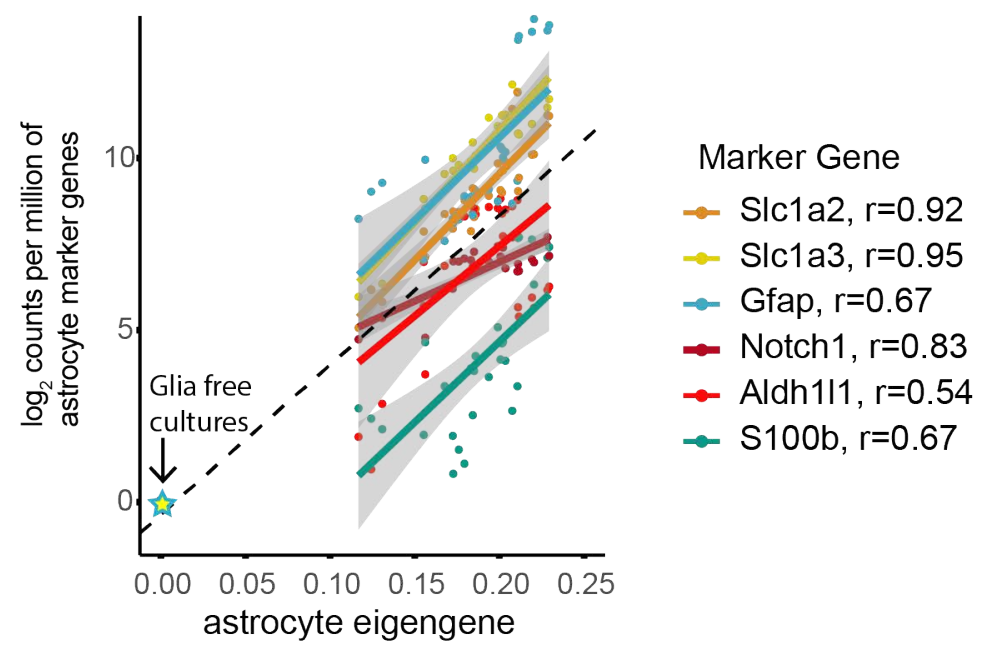

D Astrocyte eigengene has a dose-dependent effect on expression of synaptic genes in neurons

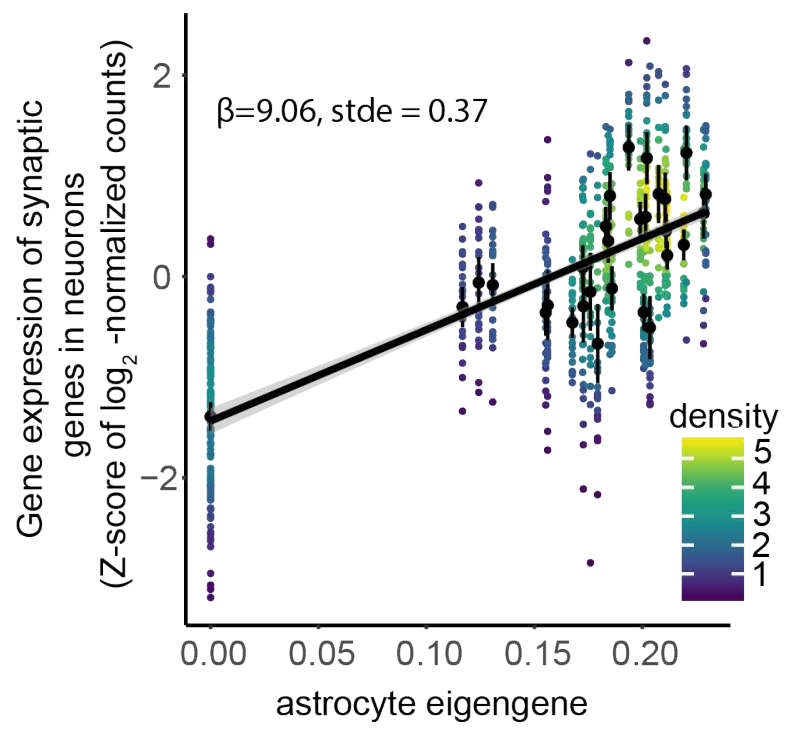

E Astrocytes induce genes with presynaptic and postsynaptic functions

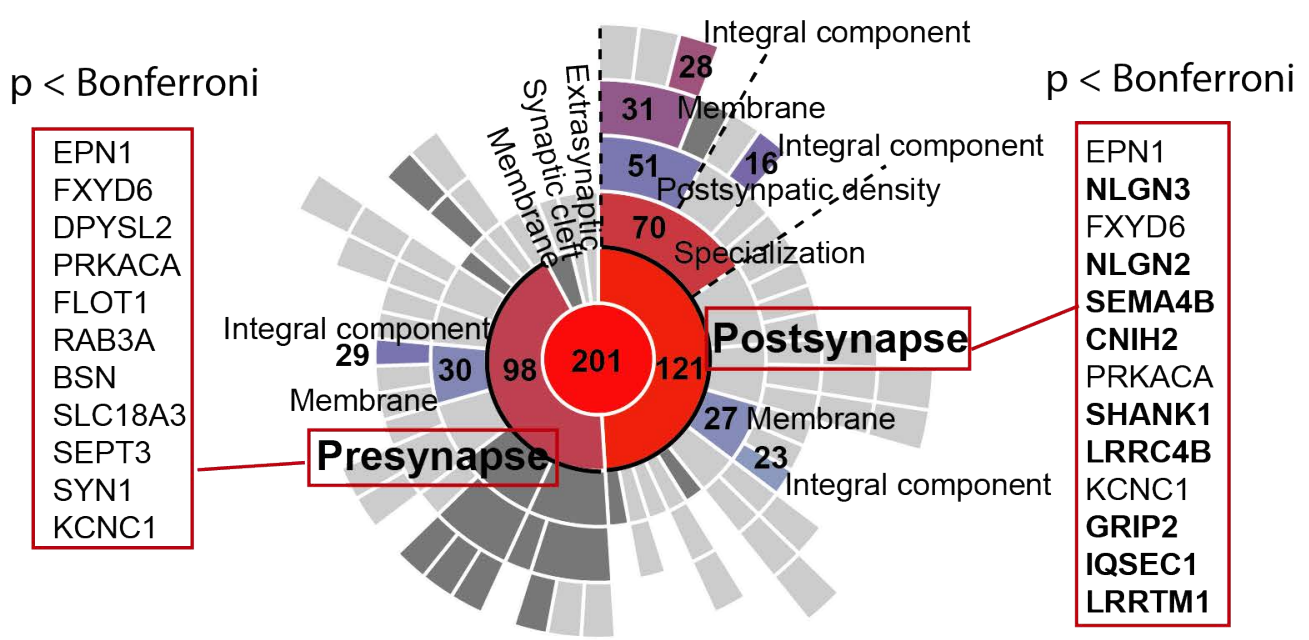

-log10 Q-value

too few genes not significant 2 3 4 5 6 $\geq 7$ 
Figure 4. Differential expression in glial cells of the neuron eigengene. A) The neuron eigengene is highly correlated with expression of astrocyte marker genes (astrocyte eigengene). B) Differential expression analysis revealed association to $123 \mathrm{glial}$ genes whose high expression is associated with neuronal maturation in coculture (orange) and 36 genes with negative association to the neuronal maturation state (blue). C) The 123 positively associated genes had the highest expression in astrocytes in the single cell atlas of adult mouse brain. The 36 negatively associated genes were uniformly expressed across glial cell types in the mouse brain atlas.

A Astrocyte eigengene and neuron eigengene are correlated $(r=0.66)$

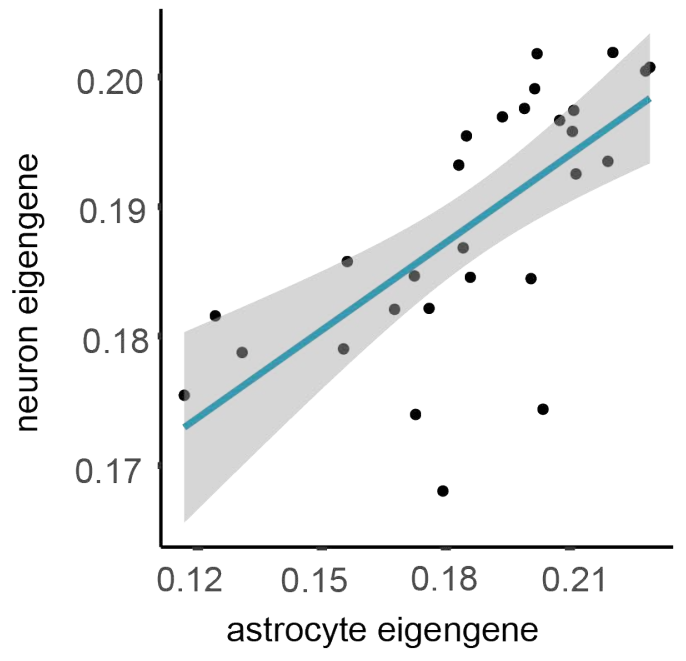

B Expression of 159 glial-genes is associated to neuronal eigengene $\left(4.3 \times 10^{-6}\right.$, Bonferroni)

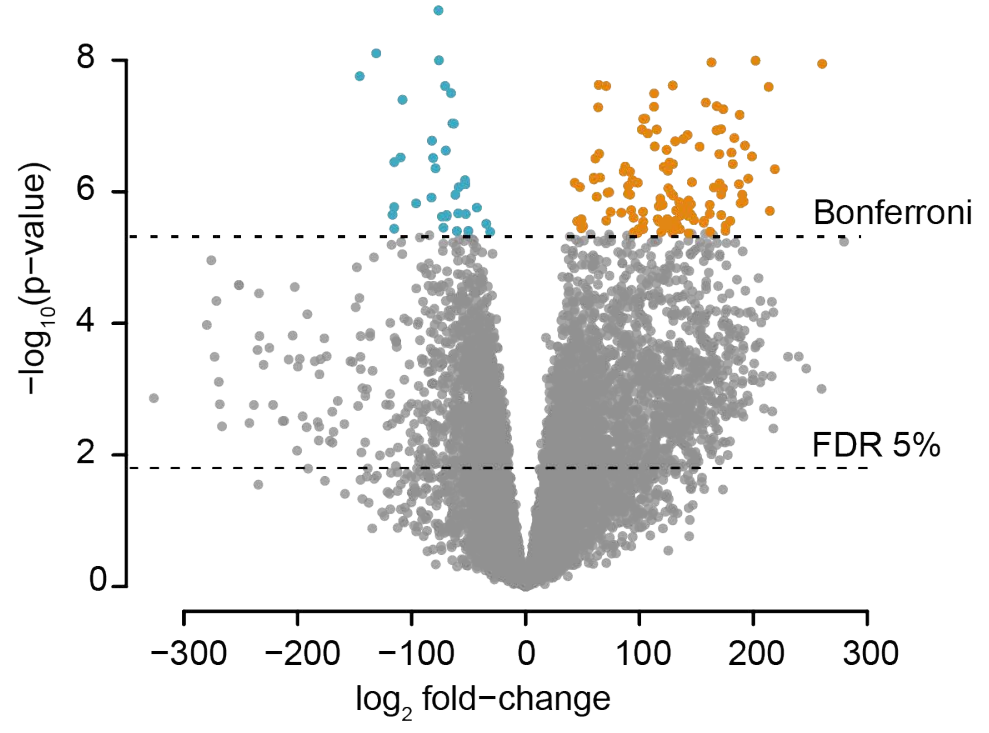

C

Glial-genes with positive association to neuron eigengene are predominantly expressed by astrocytes in adult mouse brain. Genes with negative effect are uniformly expressed.

123 genes with positive association

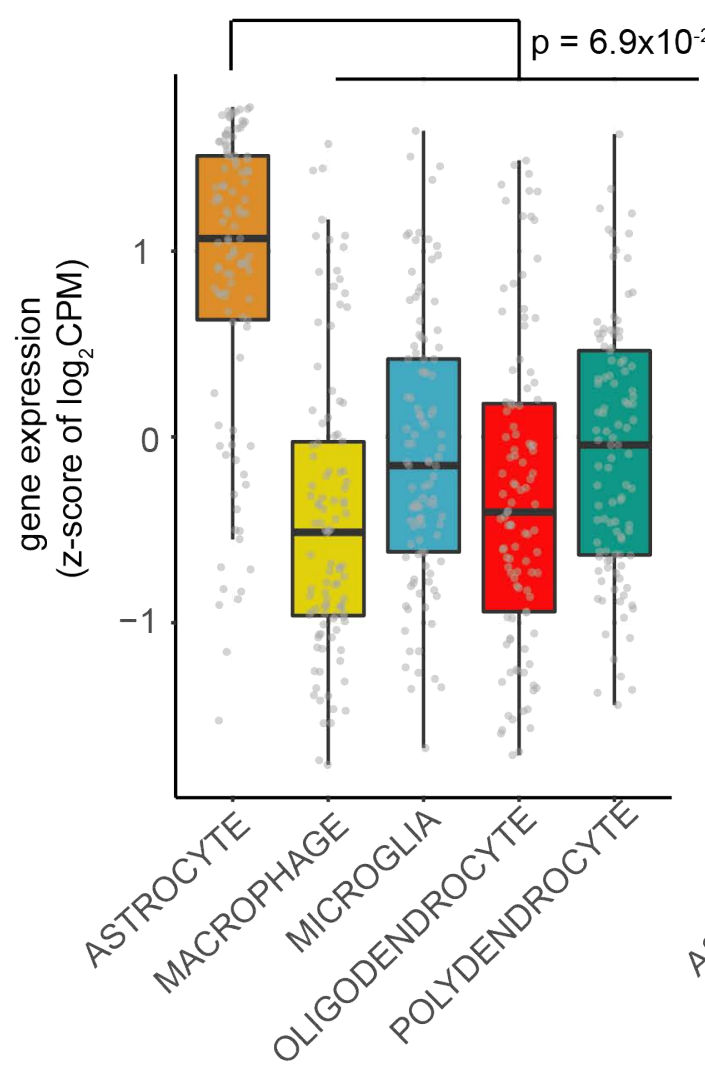

36 genes with negative association

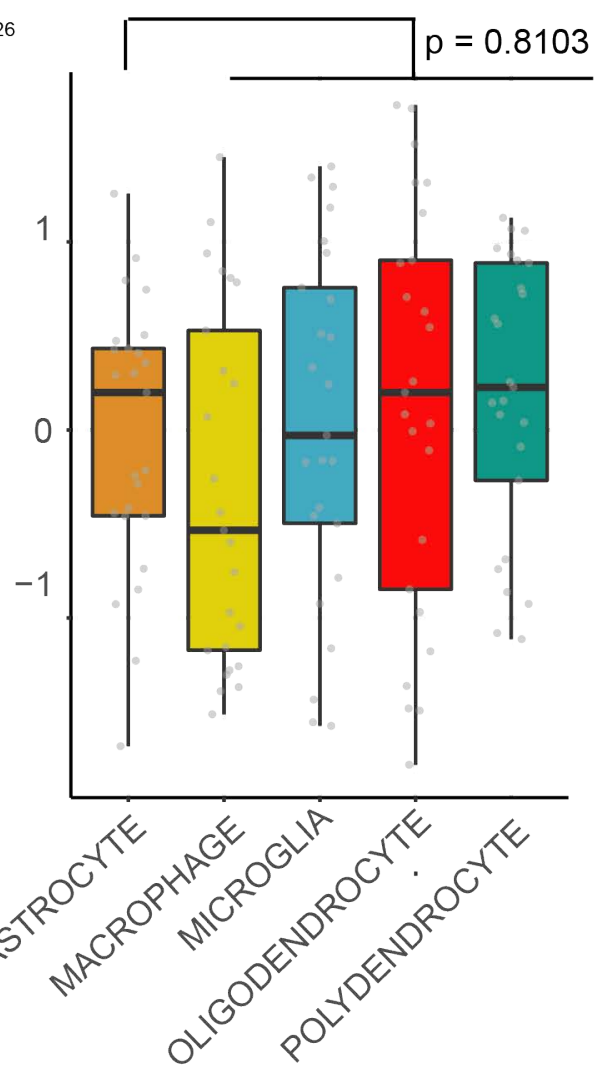


Figure 5. Glial genes with synaptic functions are associated with the neuron maturation state. A) The 159 glial geneshave synaptic functions in SynGO. B) Association of expression of glial genes with neuron eigengene. Postsynaptic genes are labeled in blue, presynaptic genes in purple and other genes are in black

A Glial genes have synaptic functions

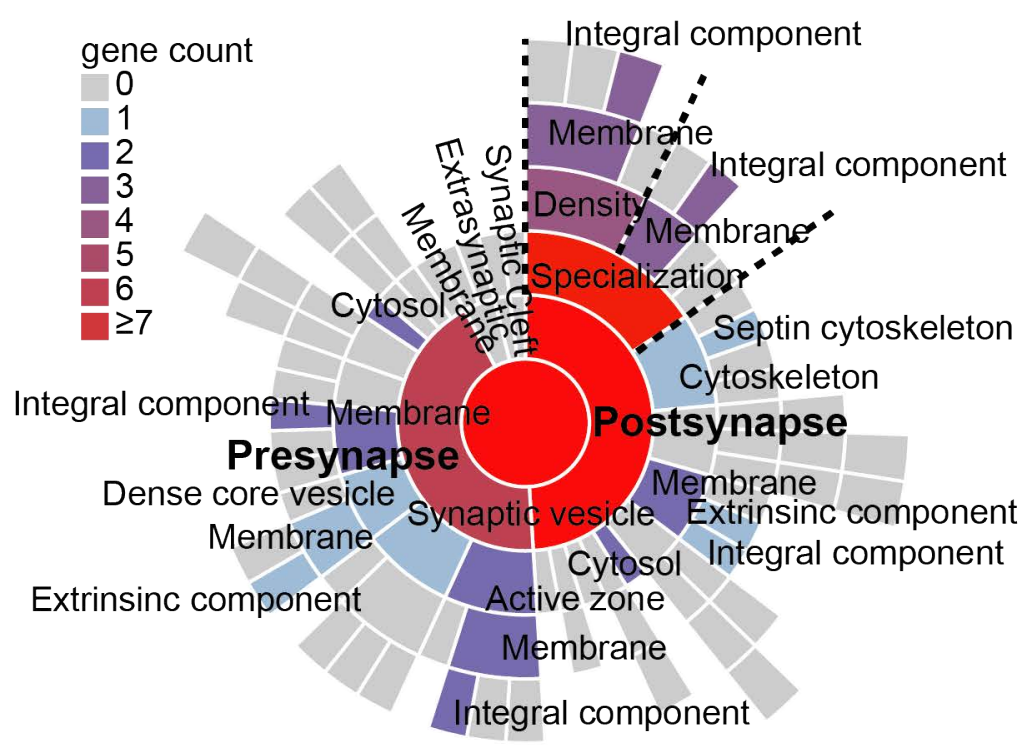

B Glial-expressed genes with synaptic functions associate with neuron geigengene values (post- and pre-synaptic, other in black)

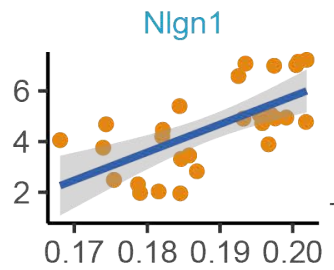

Lrrc4

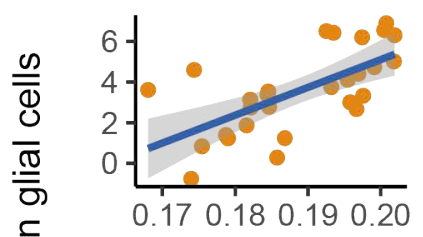

Lhfpl4

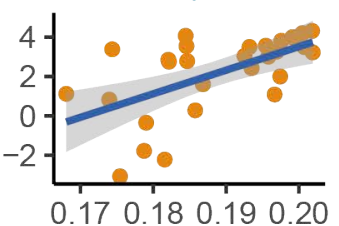

Cadps

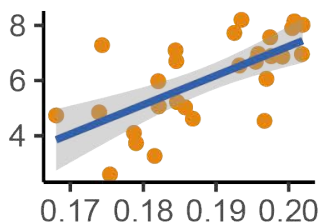

Nlgn3

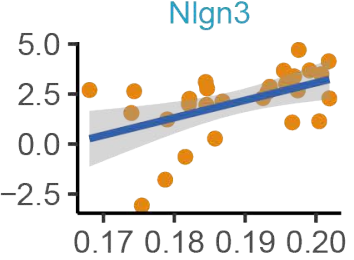

Psd2

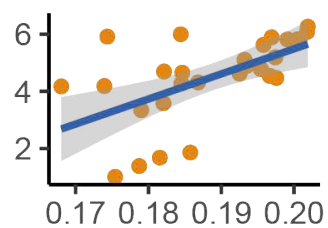

Eif4ebp1

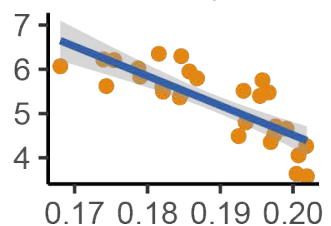

Unc13b

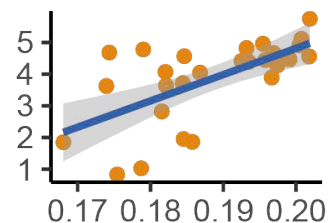

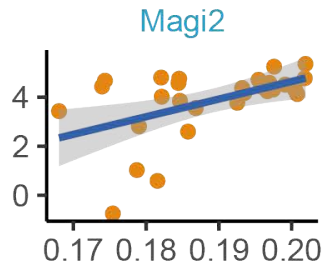

Dmd

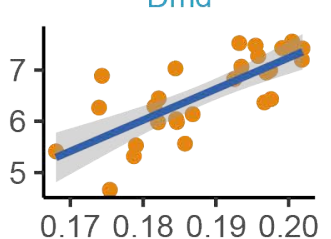

Gria1

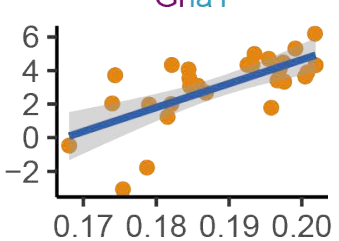

Trim9

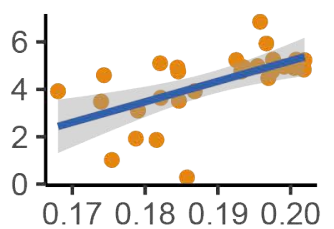

Lrrc4b

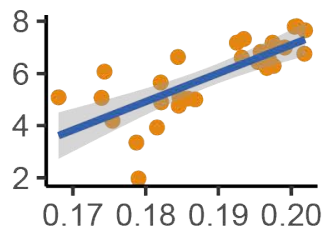

Kcnb1

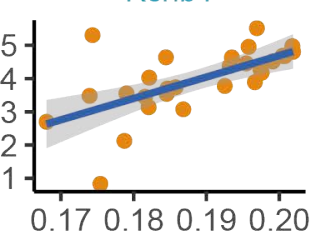

Nrxn1

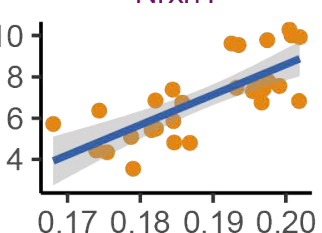

Sorcs 1

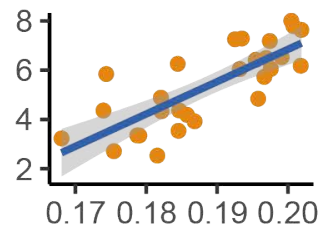

Aldoc

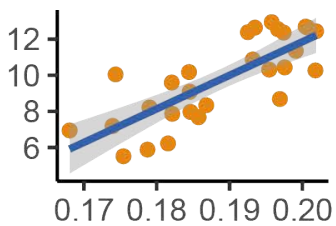

Abi2

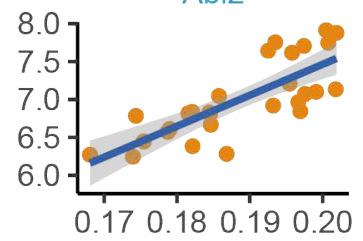

Prkcb

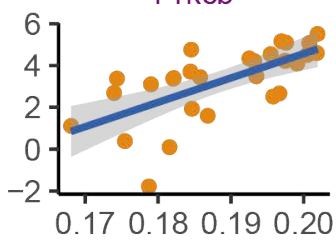

Lrp4

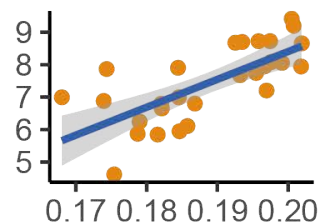

neuron eigengene 
Figure 6. Glia-neuron interactions induce genes that associate with schizophrenia. A\&B) Gene-set association analysis for four CNS traits in neurons (A) and glial cells (B). P-values for gene-set associations for each trait and gene set is indicated in the plot. C) Gene-wise associations for 123 glial genes whose high expression induce synaptic gene programs in neurons. The heat-map coloring indicates the standardized z-score of gene-wise p-values from MAGMA. The genes are ordered by the strength of association (z-score value) to schizophrenia. Synaptic genes are colored in red. Genes with direct evidence through coding variants and fine mapping in schizophrenia are indicated by arrows. Genes located in genome-wide significant loci in PGC3 for schizophrenia are highlighted by blue boxes.

A Astrocytes induce transcripts encoded by genes associated with schizophrenia in neurons

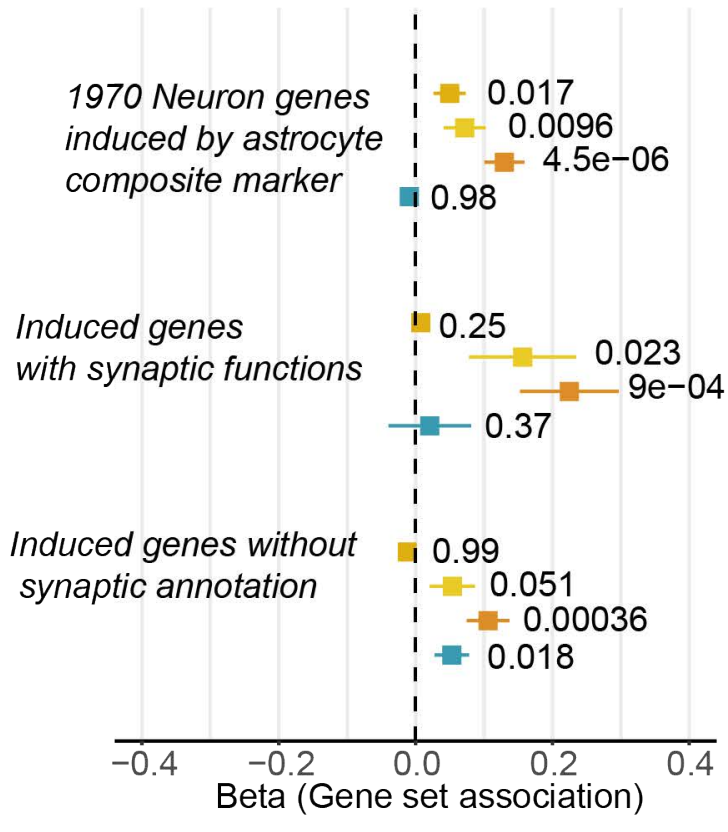

B Glial-expressed genes promoting neuronal maturation associate with schizophrenia

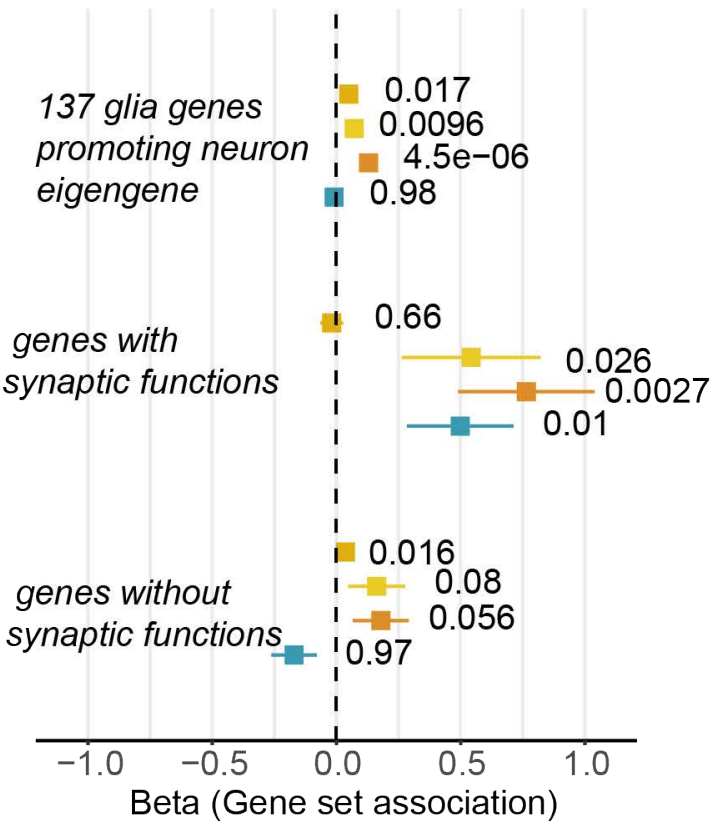

C Gene-wise association of 123 glial genes to CNS traits in ranked order for strength of association to schizophrenia

\section{Strongest SCZ \\ association}
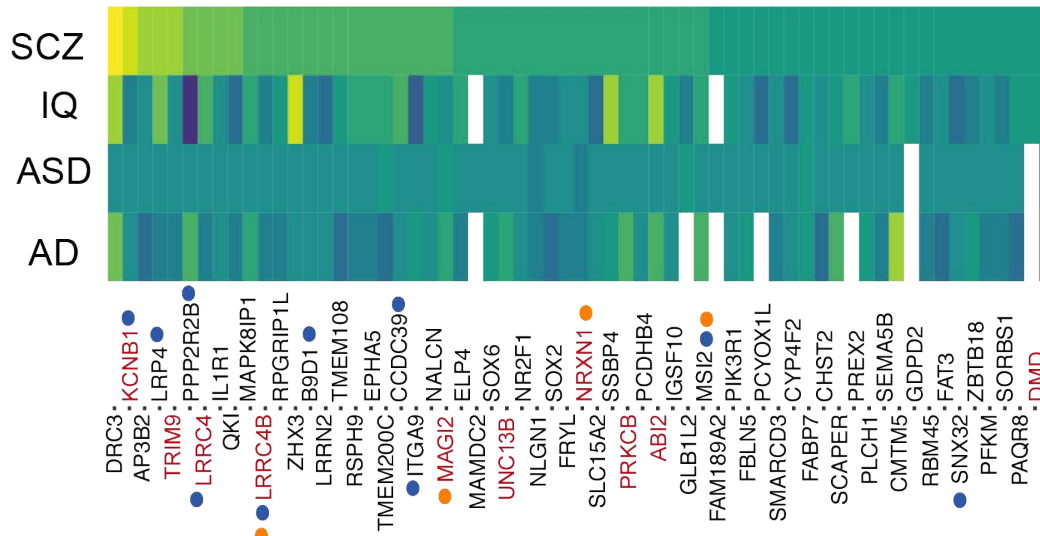

Gene-wise association
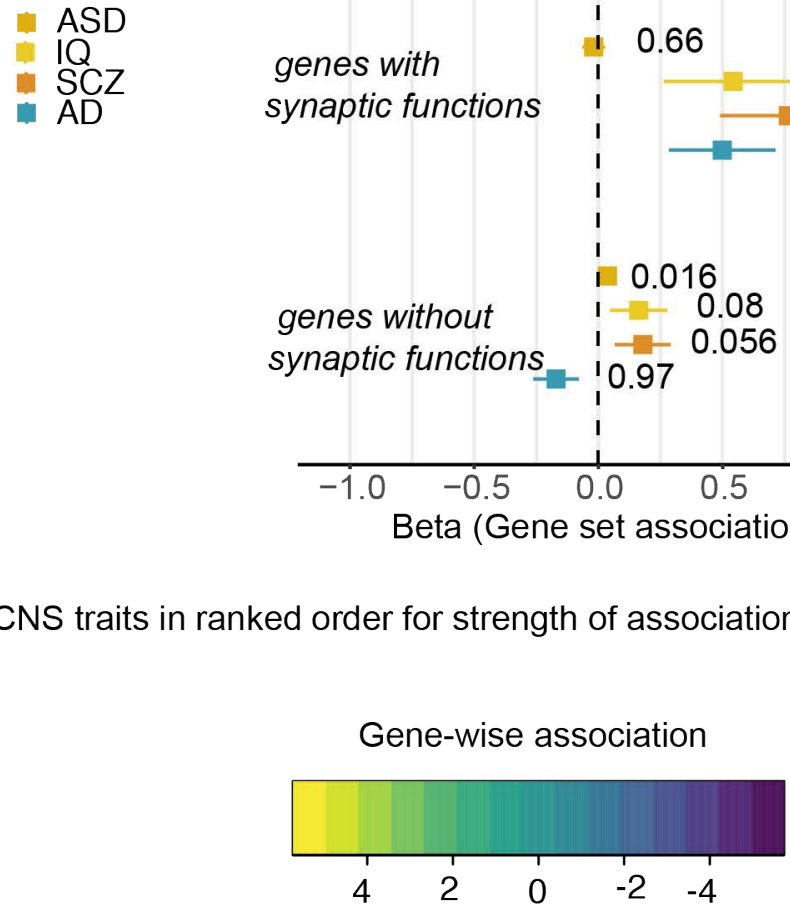

\section{Weakest SCZ association} (1)

\section{.}


Supplementary Figure 1. PCA and canonical marker gene expression in day 28 neurons.

A) PCA of RNASeq data from day 28 neurons aligned to mixed reference genome. B) Canonical marker gene expression for pluripotency, neuronal progenitor cells and neuronal identity at different stages of differentiation and culture conditions.

PC1 \& PC2 of NPCs and neurons aligned to mixed reference genome

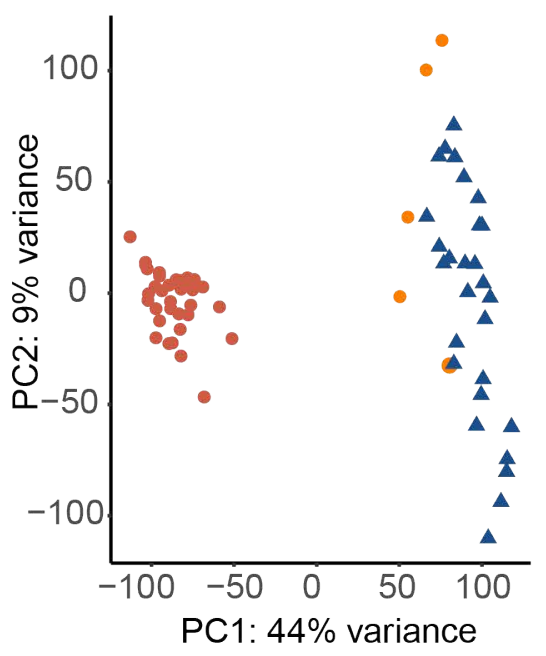

B

Expression of canonical marker genes for pluripotency, neuronal progenitors and neurons for different cell stages and culture condtions

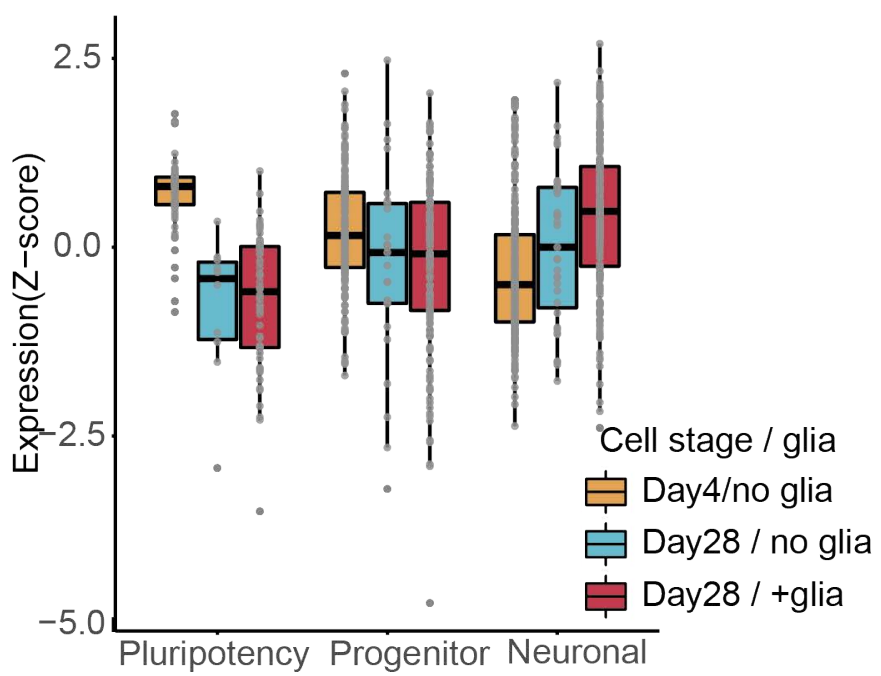


Transcriptome-wide signficantly induced genes are enriched for functions in neuronal development and synapse

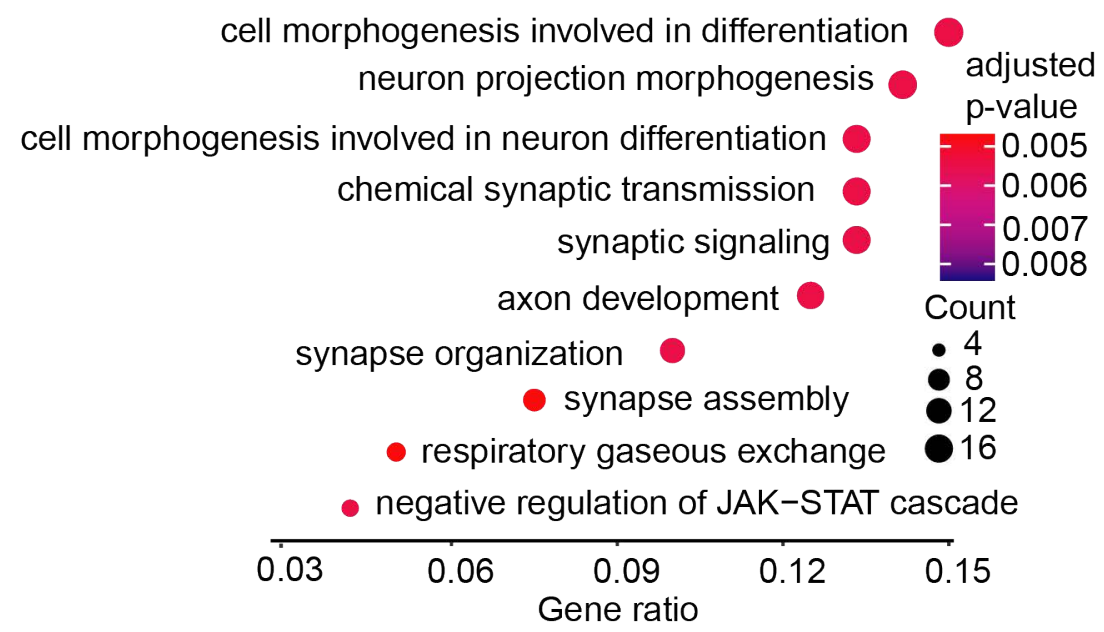

